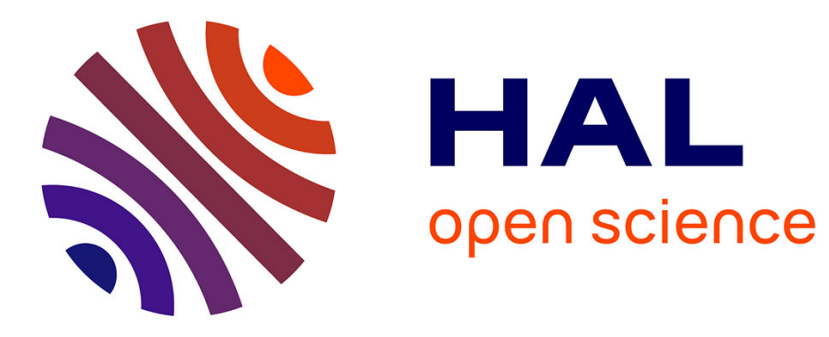

\title{
Two cGAS-like receptors induce antiviral immunity in Drosophila
}

Andreas Holleufer, Kasper Grønbjerg Winther, Hans Henrik Gad, Xianlong

Ai, Yuqiang Chen, Lihua Li, Ziming Wei, Huimin Deng, Jiyong Liu, Ninna

Ahlmann Frederiksen, et al.

\section{To cite this version:}

Andreas Holleufer, Kasper Grønbjerg Winther, Hans Henrik Gad, Xianlong Ai, Yuqiang Chen, et al. Two cGAS-like receptors induce antiviral immunity in Drosophila. Nature, 2021, 597 (7874), pp.114-118. 10.1038/s41586-021-03800-z . hal-03340060

\section{HAL Id: hal-03340060 https://hal.science/hal-03340060}

Submitted on 9 Sep 2021

HAL is a multi-disciplinary open access archive for the deposit and dissemination of scientific research documents, whether they are published or not. The documents may come from teaching and research institutions in France or abroad, or from public or private research centers.
L'archive ouverte pluridisciplinaire HAL, est destinée au dépôt et à la diffusion de documents scientifiques de niveau recherche, publiés ou non, émanant des établissements d'enseignement et de recherche français ou étrangers, des laboratoires publics ou privés. 


\section{Two cGAS-like receptors induce antiviral immunity in Drosophila}

Andreas Holleufer ${ }^{1 *}$, Kasper Grønbjerg Winther ${ }^{2 *}$, Hans Henrik Gad ${ }^{1 *}$, Xianlong $\mathrm{Ai}^{3}$, Yuqiang Chen ${ }^{3}$, Lihua $\mathrm{Li}^{3}$, Ziming $\mathrm{Wei}^{3}$, Huimin Deng ${ }^{3}$, Jiyong Liu ${ }^{3}$, Ninna Ahlmann Frederiksen ${ }^{1}$, Bine Simonsen ${ }^{1}$, Line Lykke Andersen ${ }^{4}$, Karin Kleigrewe ${ }^{5}$, Louise Dalskov ${ }^{1}$, Andreas Pichlmair ${ }^{4,6}$, Hua Cai ${ }^{3 * *}$, Jean-Luc Imler ${ }^{2,3 * *}$ and Rune Hartmann ${ }^{1 * *}$.

\section{Affiliations:}

${ }^{1}$ Department of Molecular Biology and Genetics, Aarhus University, Aarhus, Denmark.

${ }^{2}$ University of Strasbourg, CNRS UPR9022, Strasbourg, France.

${ }^{3}$ Sino-French Hoffmann Institute, School of Basic Medical Science, Guangzhou Medical University, Guangzhou, China.

${ }^{4}$ Institute of Virology, Technical University of Munich, Munich, Germany.

${ }^{5}$ Bavarian Center for Biomolecular Mass Spectrometry, Technical University of Munich, Freising, Germany

${ }^{6}$ German Center for Infection Research (DZIF), Munich partner site, Munich, Germany

* These authors contributed equally to this work.

**Corresponding authors: chjorbe@hotmail.com (H.C.); jl.imler@ibmc-cnrs.unistra.fr

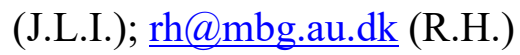


In mammals, cGAS produces the cyclic dinucleotide (CDN) 2'3'-cGAMP in response to cytosolic DNA and this triggers an antiviral immune response. cGAS belongs to a large family of cGAS/DncV-like nucleotidyltransferases, present in both prokaryotes ${ }^{1}$ and eukaryotes $^{2-5}$. In bacteria, these enzymes synthesize a range of cyclic oligonucleotide and have emerged as important regulators of phage infections ${ }^{6-8}$. Here, we identify two novel cGAS-like receptors (cGLRs) in the insect Drosophila melanogaster. We show that to infection with RNA or DNA viruses. cGLR1 is activated by dsRNA to produce the novel CDN 3' 2 '-cGAMP whereas cGLR2 produces a combination of 2'3'-cGAMP and 3'2' cGAMP in response to a yet unidentified stimulus. Our data establish cGAS as the founding member of a family of receptors sensing different types of nucleic acids and triggering immunity through production of CDNs beyond 2'3'-cGAMP.

Insects represent $65 \%$ of all living animal species and host a wide diversity of viruses, yet we know relatively little about how viral infections are recognized within this group of animals. The common perception of antiviral immunity in insects has so far been dominated by the extensive characterization of the RNA interference (RNAi) pathway ${ }^{9}$. However, transcriptional responses to virus infections have also been identified in the fruit fly Drosophila melanogaster, several genera of mosquitos and other insects. The Toll and immune deficiency (IMD) pathways, as well as the JAK-STAT pathway, have been proposed to participate in antiviral defenses, although the exact mechanisms are poorly described ${ }^{10}$. In addition, we and others recently reported that the signaling adapter Sting participates in antiviral immunity in D. melanogaster as well as the silkworm Bombyx mori ${ }^{11-13}$. However, how virus infections are recognized to induce these pathways in insects remains unknown and so far the only identified sensor for viral nucleic acids, a hallmark of viral infection, is the DEX/DH box helicase Dicer-2, which detects double stranded (ds) RNA and activates the RNAi pathway ${ }^{14}$. In mammals, STING binds a variety of cyclic dinucleotides (CDN) $)^{15,16}$ but is most strongly activated by the second messenger 2'3'-cGAMP, which is produced by the enzyme cGAS ${ }^{2-5,17-20}$. We recently showed that injection of 2'3'-cGAMP into the body cavity of adult flies induces potent Sting signaling and triggers a broad antiviral protection, suggesting that pattern recognition receptors (PRRs) with CDN synthase activity sense viral infection in flies ${ }^{21}$.

The D. melanogaster gene CG7194 was described as a homologue of $\mathrm{cGAS}^{22}$, but so far no enzymatic activity has been described and CG7194 deficient flies appear to have a normal 
antiviral immune response ${ }^{23}$. We identified four hitherto undescribed D. melanogaster gene products related to CG7194 and cGAS, of which two, CG12970 and CG30424, harbored a conserved active site compatible with CDN synthase activity (Fig. 1a). In the case of CG30424, the annotated start methionine is situated in the active site and would be incompatible with the fold of a CDN synthase. However, we identified an upstream in-frame Interestingly, all candidates lack the characteristic Zn-finger motif as well as the secondary binding site found in cGAS, suggesting that their presumed interaction with nucleic acids must differ significantly (Extended Data Fig. 1). Thus, the D. melanogaster genome encodes two proteins with putative CDN synthase activity besides CG7194 and based on the data presented below, we named them cGAS-like receptor 1 and 2 (cGLR1 and cGLR2, encoded by $C G 12970$ and $C G 30424$, respectively).

To test if the identified CDN synthases can activate signaling, we used the macrophage-like $\mathrm{S} 2$ cell line and a luciferase reporter system based on the promoter of the Sting gene since activation of Sting induces its own transcription ${ }^{11}$. Expression of human cGAS in S2 cells led to activation of the Sting reporter and this activation was abolished by mutation of the active site (Fig. 1b, Extended Data Fig. 2), agreeing with our previous discovery that transfection of 2'3'-cGAMP in S2 cells triggers Sting signaling ${ }^{21}$. Expression of cGLR1 and cGLR2 resulted in significant upregulation of the Sting reporter, and mutation of their predicted active site abrogated this activity. Despite exhibiting the highest homology to human cGAS, CG7194 did not yield any detectable activity in this assay. We conclude that we have identified two cGAS-like enzymes that could function as PRRs sensing viral infection in D. melanogaster.

To confirm these results in vivo, we generated transgenic flies expressing wild-type or catalytically inactive mutants of cGLR1 and cGLR2 using the heat-inducible Gal4Gal80/UAS system ${ }^{24}$. We confirmed that both genes were overexpressed (Extended Data Fig. $3 \mathrm{a}, \mathrm{b})$ at $29^{\circ} \mathrm{C}$ and that this did not have a major effect on the viability of the flies (Extended Data Fig. 3e). Expression levels of Sting and the Sting-regulated genes ( $\mathrm{Srg}$ ) 1, 2, and $3^{21}$ were all significantly upregulated in flies overexpressing wild-type cGLR2 compared to the catalytically inactive mutant (Fig. 1c, d and Extended Data Fig. 3c, d). Similarly, overexpression of cGLR1 led to a significant upregulation of Sting, Srg2, and Srg3, whereas the trend observed for $\mathrm{Srg} 1$ did not reach significance. Finally, overexpression of cGLR1 and cGLR2 led to a significant reduction of vesicular stomatitis virus (VSV) replication compared to the catalytically inactive mutants (Fig. 1e). Overexpression of cGLR2 also reduced 
replication of Drosophila C virus (DCV), a natural Drosophila pathogen, whereas the slight reduction observed with cGLR1 was not significant for this virus (Fig. 1f). However, transgenic flies expressing the wild-type versions of cGLR1 and cGLR2, but not the catalytically inactive mutants, exhibited a striking increase in survival following infection with DCV (Fig. 1g). We conclude that cGLR1 and cGLR2 can initiate a transcriptional response in vivo, associated with protection against virus infection.

To test if signaling by cGLR1 and cGLR2 depends upon Sting, we introduced a frameshift deletion in the Sting gene of S2 cells (Extended Data Fig. 4d, e). Activation of the Sting reporter by cGLR1, cGLR2 and cGAS was abolished in the knockout cells but was rescued when co-transfecting with Sting (Extended Data Fig. 4a, b). Thus, Sting is required for cGLR1- and cGLR2-mediated signaling. Sting drives the expression of antiviral genes through Relish, a member of the NF- $\mathrm{BB}$ family of transcription factors ${ }^{11,21}$. Mutation of the Relish binding site within the Sting promoter abolished the response to cGLR1 and cGLR2, connecting these receptors to NF- $\kappa \mathrm{B}$-dependent transcription (Extended Data Fig. 4c). This is in contrast to the mammalian system, where members of the interferon regulatory factor (IRF) family of transcription factors mediate most of the antiviral effect ${ }^{25,26}$, whereas NF- $\kappa \mathrm{B}$ is thought to primarily drive expression of pro-inflammatory cytokines.

To show that cGLR1 and cGLR2 are required for initiating an antiviral immune response in flies, we generated cGLR1 or cGLR2 knockout (KO) flies by CRISPR/Cas9 mutagenesis (Extended Data Fig. 5). Next, we generated cGLR1 and cGLR2 double KO flies by recombination (denoted cGLR1/2 KO). All flies were viable and resisted the stress of a buffer injection (Extended Data Fig. 6a). Upon infection with DCV cGLR1 KO but not cGLR2 KO flies exhibited reduced survival whereas the cGLR1/2 KO flies had a significantly lower survival than either of the two single KO flies (Fig. 2a). In agreement with the survival data, we observed a trend for increased DCV replication in cGLR1 KO flies compared to control and cGLR2 KO flies, which became statistically significant in the cGLR1/2 double KO flies (Fig. 2c). Finally, expression of Sting-regulated genes was reduced in both cGLR1 KO and cGLR1/2 KO flies (Fig. 2d and Extended Data Fig 6b, c, d). Next, we infected both cGLR1 and cGLR2 KO flies with Kallithea virus $(\mathrm{KV})$, a large DNA virus and also a natural Drosophila pathogen, and observed reduced survival in both KO flies (Fig. 2b and Extended Data Fig. 7a). However, this was not accompanied by a significant increase in viral replication in either KO flies (Fig. 2e). As previously reported ${ }^{27}$, KV did induce expression of Sting-regulated genes and this induction was lost in cGLR1 KO flies. Intriguingly, the 
induction of Srg3, but not Sting, Srg1, and Srg2, was affected in cGLR2 KO flies (Fig. 2f and Extended Data Fig 7b, c, d). Similar to what was observed for DCV, cGLR1/2 KO flies exhibited a more severe phenotype upon KV infection than single KO flies (Extended Data Fig. 7e, f, g). We also tested infection by VSV and invertebrate iridescent virus 6 (IIV6), but neither of them showed any overt phenotype for survival and viral load (Extended Data Fig. 8 and 9). However, we observed that one of the marker genes, Srg3, was induced by VSV and IIV6 infection in control and cGLR1 KO flies, but not in the cGLR2 KO flies (Extended Data Fig. 8g and 9g). We conclude that both cGLR1 and cGLR2 are required for survival of flies upon infection with two natural viral pathogens of Drosophila, DCV and KV.

Next, we expressed cGLR1, cGLR2 or cGAS alone or together with human STING in the human cell line HEK293T, which lacks endogenous expression of STING. Here, cGLR2 induced signaling via STING to a level comparable to that of cGAS (Fig. 3a and Extended Data Fig. 10a). Furthermore, this depended upon the active site of cGLR2, suggesting that cGLR2 produces a CDN capable of activating STING (Extended Data Fig. 10b, c). In contrast, cGLR1 did not activate STING signaling in HEK293T cells, (Fig. 3a and Extended Data Fig. 10a). To identify the CDN synthetized by cGLR2, we isolated the nucleotidecontaining fraction from HEK293T cells expressing cGLR2 and then subjected this extract to analysis by mass spectrometry. Interestingly, Slavik et al. demonstrate that cGLR1 produces 3'2'-cGAMP ${ }^{28}$. We therefore established a protocol to detect both 2'3'-cGAMP and 3'2'cGAMP, which revealed that the two CDNs are synthesized in approximately equivalent amounts by cGLR2 in HEK293T cells (Fig. 3b and Extended Data Fig. 10d, e, f). Both 2'3'cGAMP and 3'2'-cGAMP induced expression of Sting and Srg3 when introduced into S2 cells. The 2'3'-cGAMP mediated induction was lower than that of 3'2'-cGAMP but the difference was not statistically significant. The two CDNs also activated endogenous STING in the human cell line HT-1080, with 2'3'-cGAMP appearing the most potent activator (Extended Data Fig. 10g, h, i). Therefore, we hypothesize that the absence of activity by cGLR1 in HEK293T cells is caused by the absence of a suitable activator. The cGLRs share similarity with oligoadenylate synthetases, a family of proteins activated by dsRNA, and thus we transfected HEK293T cells first with cGLR1 and then poly(I:C), which led to activation of cGLR1 (Fig. 3c). To confirm that cGLR1 is indeed activated by dsRNA, we expressed the protein in $E$. coli, purified it and then performed an in vitro activity assay as described by Kranzusch and colleagues ${ }^{28}$. This showed that cGLR1 produces 3'2'-cGAMP and minor amounts of 2'3'-c-diAMP upon stimulation with either poly(I:C) or a 100 base pair long 
dsRNA produced by T7 transcription, but not with a similar 100 base pair long dsDNA (Fig. 3d and Extended Data Fig. 11a, b). We also attempted to purify cGLR2 but only achieved poor quality protein preparations that did not display any activity. assume that these cells produce a suitable activator. When performing transfections, the transfected DNA can act as a ligand activating cGAS and potentially cGLR2. To circumvent this issue, we generated HEK293T cells stably expressing either cGAS or cGLR2 by retroviral transduction. cGAS only produced 2'3'-cGAMP upon transfection with DNA (Fig. 3e). However, cGLR2 produced both 3'2'-cGAMP and 2'3'-cGAMP, regardless of the presence of cytosolic DNA supplied by transfection. Thus, we conclude that the ligand activating cGLR2 is not dsDNA. As demonstrated above, cGLR1 is inactive in HEK293T cells unless dsRNA is provided by transfection, indicating that dsRNA is not present in our HEK293T cells. Thus, the activity of cGLR2 in HEK293T cells cannot be explained by the presence of either dsRNA or dsDNA. It is possible that cGLR2 is constitutively active and to address this, we mutated residues in the putative nucleic acid-binding domain (K256E/R267E and K256E/R271E). Those residues are conserved in cGAS and oligoadenylate synthetases (OAS) and are known to be critical for DNA- and RNA-induced activation of these enzymes $^{3,29}$. Mutation of those residues led to significant loss of activity in S2 cells and a complete loss of activity in HEK293T cells (Fig. 3f, Extended Data Fig. 11c, d). In summary, our data suggest that cGLR2 requires allosteric activation by a nucleic acid.

To conclude, we have identified two novel PRRs, cGLR1 and cGLR2, which orchestrate a Sting and NF- $\kappa \mathrm{B}$ dependent antiviral immune response in D. melanogaster. Several primordial functions of Sting have been suggested, including sensing of bacteria through recognition of bacterially derived $\mathrm{CDNs}^{23}$ or autophagy ${ }^{12,30}$. Our data suggest that a cGLRSting-NF- $\kappa \mathrm{B}$ axis was present already during early metazoan evolution. They further reveal that the production of CDNs by metazoan enzymes is not limited to 2'3'-cGAMP. Different CDNs could lead to diverse signaling outcomes, interact with alternative receptors ${ }^{31}$ or have different stability towards degradation by both host and viral encoded degrading enzymes, e.g. poxins ${ }^{32-34}$. Future studies are needed to determine the biological role of different cGAMP isomers.

The cGLRs are the first reported class of antiviral PRRs responsible for inducing a transcriptional response in insects. One striking difference between the cGLRs and cGAS is the specificity in activation. Whereas cGAS contains a Zn-finger motif inserted early in 
vertebrate evolution, which confers specificity for DNA, the cGLRs have a nucleic acidbinding groove more akin to OAS. We show that cGLR1 is activated by dsRNA and this is consistent with our in vivo data, which reveal a strong susceptibility of cGLR1 mutant flies to

Acknowledgements. The authors wish to thank Kailey Slavik and Philip Kranzusch for discussing and sharing information prior to publication, Peter E. Andersen for help with generating Sting knockout S2 cells, Darren Obbard for providing Kallithea virus and C. Meignin, J. Marques, J. Schneider and G. Haas for helpful discussions. R.H was supported by grants from the Novo Nordisk Foundation (NNF17OC0028184) and the Danish Council for Independent Research (4183-0032B and 0135-00338B). J.L.I. was supported by Agence Nationale de la Recherche (ANR-17-CE15-0014), Investissement d'Avenir Programs (ANR10-LABX-0036, ANR-11-EQPX-0022), Institut Universitaire de France and the Chinese National Overseas Expertise Introduction Center for Discipline Innovation (Project "111" (D18010)). H.C was supported by the Natural Science Foundation (32000662) and the Foreign Experts Program (2020A1414010306). A.P. was supported by an ERC consolidator grant (ERC-CoG ProDAP, 817798) and grants from the German Research Foundation (PI 1084/5, TRR179, and TRR237). 
Author contributions. A.H., K.G.W., H.H.G., H.C., J.L.I., and R.H. conceived and designed experiments for this study. A.H., K.G.W., H.H.G., X.A., Y.C., L.L., Z.W., H.D., J.L., N.A.F., B.S., L.L.A., K.K., L.D. and H.C. performed experiments. A.H., H.H.G., and H.C. created the figures. H.C., A.P., J.L.I., and R.H. supervised the study. A.H., J.L.I. and R.H. wrote the original draft of the manuscript and all authors participated in reviewing and editing it.

Competing interests. The authors declare that they have no competing interests.

\section{Methods}

\section{Alignment of cGAS-like proteins}

The five cGAS-like candidate proteins from D. melanogaster, CG12970 (UniProt: A1ZA55), CG30424 (UniProt: A8DYP7), CG4746 (UniProt: Q9U3W6), CG4766 (UniProt: Q9Y106), and CG7194 (UniProt: Q9VSH0) were aligned with porcine OAS1 (UniProt: Q29599), human OAS1 (UniProt: P00973-1), human cGAS (UniProt: Q8N884-1), porcine cGAS (UniProt: I3LM39), murine cGAS (UniProt: Q8BSY1) using CLC Main Workbench 7.7.2 (QIAGEN). Structurally homologous secondary structure elements of the mammalian nucleotidyltransferases were used as alignment fix points.

\section{Cell lines}

HEK293T and HT-1080 cells were obtained from ATCC (CRL3216) and the German Collection of Microorganisms and Cell Cultures (ACC 315), respectively, whereas HEK293T/MAVS KO cells were a kind gift from Veit Hornung (Gene Center Munich). All mammalian cells were cultured in Dulbecco's modified Eagle medium (DMEM) (SigmaAldrich) supplemented with 10\% fetal bovine serum (FBS) (Biowest), $100 \mathrm{U} \mathrm{ml}^{-1}$ penicillin (Sigma-Aldrich) and $100 \mu \mathrm{g} \mathrm{ml}^{-1}$ streptomycin (Sigma-Aldrich) at $37^{\circ} \mathrm{C}$ and $5 \% \mathrm{CO}_{2}$. Schneider 2 (S2) cells were obtained from the German Collection of Microorganisms and Cell Cultures (ACC 130) and cultured in Schneider's Drosophila Medium (Biowest) supplemented with $10 \% \mathrm{FBS}, 100 \mathrm{U} \mathrm{ml}^{-1}$ penicillin (Sigma-Aldrich) and $100 \mu \mathrm{g} \mathrm{ml}$ streptomycin (Sigma-Aldrich) at $27^{\circ} \mathrm{C}$.

\section{Fly lines}

Fly stocks were raised on standard cornmeal agar medium at $25^{\circ} \mathrm{C}$. All fly lines used in this study were free of Wolbachia. The drivers used were [actin5C-Gal4/CyO; tubulin- 
Gal80 5 ts $/ \mathrm{TM}$, Tb]. Transgenic lines for expression of GFP and $\beta$-galactosidase (P $\{$ UASGFP.nls 8 line (BDSC \#4776) and P\{UAS-LacZ.Exel\}2 (BDSC \#8529)) were obtained from the Bloomington Drosophila Stock Center. For the UAS transgenic lines of wild-type and AFA mutant versions of cGLR1 and cGLR2, the corresponding versions of the cDNAs (RD isoform in the case of cGLR2) were cloned into pUAST-attB vectors. The resulting plasmids flies (BDSC \#24749). Individual males from the injected embryos were then crossed with flies containing the third chromosome balancers $T M 6 B, T b / T M 3, S b$ to establish balanced stocks. $c G L R 1$ and $c G L R 2$ knockout flies were generated by CRISPR-Cas mediated mutagenesis. Briefly, sgRNA encoding pUAST-attB plasmids were injected into embryos of $y^{l} M\left\{\right.$ vas-int.Dm\}ZH-2A $w^{*} ; M\{3 x P 3-R F P . a t t P\} Z H-86 F b$ (BDSC\#24749) flies. The resulting transgenic flies were then crossed with $y^{1} w^{1118}$; attP2\{nos-Cas9\}/TM6C, Sb $\mathrm{Tb}[y+]$ flies obtained from the National Institute of Genetics. Individual males from the F1 were then crossed with $y w ; B c, G l a / C y O$ flies to establish stocks from the $C y O$ progeny. This progeny was then scored for mutations by Sanger sequencing (Guangzhou IGE Biotechnology). cGLR1 (2R: 52C8) and cGLR2 (2R: 60E11) knockout flies were isogenized to the DrosDel $w^{1118}$ isogenic background to reduce the genetic background effects. For each line, the nonmutated chromosomes were replaced using balancer chromosomes $\left(w^{1118}\right.$; If/CyO; TM3/TM6 and $w^{1118} ; \mathrm{If} / \mathrm{CyO}$ ) whereas the mutations were recombined to the respective DrosDel $w^{1118}$ isogenic chromosome for seven generations. We confirmed that the isogenized lines retained the mutation of interest by DNA sequencing. $c G L R 1$ and $c G L R 2$ double knockout flies were generated by crossing the two single knockout flies. Females from the progeny were crossed with $w^{1118}$; If $/ C y O$ to establish the stocks from the $C y O$ progeny. Presence of the double mutation upon recombination on the right arm of the second chromosome in this progeny was established by DNA sequencing. All the crossing schemes and detailed injection protocols are available upon request.

\section{Plasmids}

cDNAs encoding human cGAS (amino acids 155-522), cGLR1, cGLR2 or CG7194 with a Cterminal V5- or triple FLAG-tags were cloned into the pAc5.1 vector for transient expression in S2 cells, the pcDNA3.1 vector for transient expression in HEK293T cells or the pCCLPGK vector for production of lentiviral particles. For expression of cGLR1 in E. coli, the cDNA was cloned into the pET-9d vector in between an N-terminal TEV protease cleavage 
site and maltose-binding protein (MBP) and a C-terminal polyhistidine-tag. The following active site mutants were generated by site-directed mutagenesis: cGAS AFA (E225A D227A), cGLR1 AFA (E71A D73A), cGLR2 AFA (E79A D81A), and CG7194 AFA (E70A D72A). cDNAs encoding human or D. melanogaster STING/Sting without or with a C-

\section{Transfection of S2 cells}

To test induction of $D$. melanogaster Sting by cGLRs, 12-well tissue culture plates were seeded with $6 \times 10^{5} \mathrm{~S} 2$ cells per well. After $24 \mathrm{~h}$, each well was transfected with $485 \mathrm{ng}$ pGL3 plasmid expressing firefly luciferase under transcriptional control of the Sting promoter, $15 \mathrm{ng}$ pAc5.1 plasmid constitutively expressing Renilla luciferase, $400 \mathrm{ng}$ pAc5.1 plasmid expressing cGAS, cGLR1, cGLR2 or CG7194 or their corresponding mutants (except Extended Data Fig. 10b where $10 \mathrm{ng}$ was used) and finally empty Ac5.1 plasmid to reach a total amount of $1 \mu \mathrm{g}$ plasmid per well. To test if the activity of cGLRs is Stingdependent, 12-well tissue culture plates were seeded with $6 \times 10^{5} \mathrm{~S} 2$ or Sting knockout S2 cells per well. After $24 \mathrm{~h}$, each well was transfected with $485 \mathrm{ng}$ pGL3 plasmid expressing firefly luciferase under transcriptional control of the Sting promoter, $15 \mathrm{ng}$ pAc5.1 plasmid constitutively expressing Renilla luciferase, $30 \mathrm{ng}$ pAc5.1 plasmid expressing Sting, $100 \mathrm{ng}$ pAc5.1 plasmid expressing cGAS, cGLR1, cGLR2 or CG7194 and finally Ac5.1 plasmid to reach a total amount of $1 \mu \mathrm{g}$ plasmid per well. All transfections of S2 cells were performed using jetOPTIMUS (Polyplus Transfection) according to the manufacturer's instructions.

\section{Transfections of HEK293T cells}

To test induction of human STING by cGLRs, 12-well tissue culture plates were seeded with $3 \times 10^{5}$ HEK293T cells per well. After $24 \mathrm{~h}$, each well was transfected with $970 \mathrm{ng}$ pGL3 plasmid expressing firefly luciferase under transcriptional control of the IFNB1 promoter, 30 ng pRL plasmid constitutively expressing Renilla luciferase, 100 ng pcDNA3.1 plasmid 

and finally empty pcDNA3.1 plasmid to reach a total amount of $2 \mu \mathrm{g}$ plasmid per well. For each well, the DNA was dissolved in $100 \mu \mathrm{l}$ DMEM and likewise $6 \mu \mathrm{g}$ polyethylenimine incubated for $20 \mathrm{~min}$ before adding it dropwise to the cells.

To test activation of cGLR1 by poly(I:C), 48-well tissue culture plates were seeded with $9 \mathrm{x}$ $10^{4}$ HEK293T/MAVS KO cells per well. After $24 \mathrm{~h}$, each well was transfected with $242.5 \mathrm{ng}$ pGL3 plasmid expressing firefly luciferase under transcriptional control of the IFNB1 promoter, $7.5 \mathrm{ng}$ pRL plasmid constitutively expressing Renilla luciferase, 10 ng pcDNA3.1 plasmid expressing STING, 240 ng pcDNA3.1 plasmid expressing cGLR1 and finally empty pcDNA3.1 plasmid to reach a total amount of $500 \mathrm{ng}$ plasmid per well. After another $3 \mathrm{~h}$, cells were transfected $150 \mathrm{ng} \operatorname{VacciGrade}^{\mathrm{TM}}$ Poly(I:C)(InvivoGen) per well. Both kind of transfection were done using Lipofectamine 2000 (Fisher Scientific) following to the manufacturer's instructions.

To test production of CDNs by cGLR2 via mass spectrometry, HEK293T cells were grown in T175 tissue culture flasks and transfected at a confluence of 60-70\%. For each flask $87.5 \mu \mathrm{g}$ pcDNA3.1 plasmid expressing EGFP, cGAS or cGLR2 was dissolved in $4.4 \mathrm{ml}$ DMEM and 262.5 $\mu \mathrm{g}$ PEI were dissolved in $4.4 \mathrm{ml}$ DMEM. The DNA and PEI was then mixed and incubated for $20 \mathrm{~min}$ before added to the cells.

\section{Transduction of HEK293T cells}

Lentiviral particles were made as previously described ${ }^{37}$. For transduction of HEK293T cells, the cells were grown in T75 tissue culture flasks and transduced at a confluence of $60-70 \%$ with filtered supernatants. The cells were then passaged for 1 week before being subjected to further experiments. Transfection of transduced cells was performed using pcDNA3.1 plasmid expressing EGFP as described above.

\section{Measuring luciferase activity of transfected cells}

Cells were lysed in $200 \mu 1$ Passive Lysis Buffer (Promega) per well and afterwards the lysates were centrifuged at $14,000 \mathrm{~g}$ for $5 \mathrm{~min}$ to remove cell debris. Firefly and Renilla luciferase activity was measured on $10 \mu \mathrm{l}$ lysate using the Dual-Luciferase ${ }^{\circledR}$ Reporter Assay System (Promega). \\ Immunoblotting}


Cell lysates were subjected to SDS-PAGE and proteins were transferred to a polyvinylidene fluoride membrane. Blotted proteins were detected with Anti-V5 Antibody (Invitrogen)(diluted 1:5,000), Anti-Actin, clone 4 (EMD Millipore)(diluted 1:5,000), AntiSting (BioGenes)(1:500), Monoclonal ANTI-FLAG® M2 (Sigma-Aldrich)(diluted 1:2,000) antibody and a Peroxidase-conjugated AffiniPure F(ab')2 Fragment Donkey Anti-Mouse IgG (JacksonImmunoResearch)(diluted 1:15,000) or Anti-Rabbit IgG, HRP-linked Antibody (Cell Signalling Technology)(diluted 1:3,000) secondary antibody. Blots were developed with SuperSignal $^{\mathrm{TM}}$ West Dura Extended Duration Substrate. The Anti-Sting antibody was raised in rabbit against the peptide HMQNKTKTIDEISN. The antibody was affinity purified from serum using the peptide and verified by immunoblotting of recombinant Sting made in Escherichia coli.

\section{Generation of Sting knockout cells by CRISPR-Cas9}

Three CRISPR RNAs targeting the first translated exon in the D. melanogaster Sting gene were cloned into the pAc-sgRNA-Casp vector. After transfection, S2 cells were grown under selection with $5 \mu \mathrm{g} \mathrm{ml} \mathrm{m}^{-1}$ puromycin for two weeks. Individual clones were isolated by limiting dilution and then screened for a non-functional Sting pathway. Knockout of Sting was verified by immunoblotting and Sanger sequencing.

\section{Mass spectrometry}

Transfected HEK293T cells were washed with PBS, trypsinized and resuspended in DMEM before being harvested by centrifugation at $800 \mathrm{~g}$ for $5 \mathrm{~min}$. The medium was carefully removed and the cells resuspended in PBS before being collected by centrifugation at $800 \mathrm{~g}$ for $5 \mathrm{~min}$. After another final washing step, the cells were lysed with $500 \mu \mathrm{L} 60 \% \mathrm{MeOH}$ and vortexed for 15 seconds. For the spike in samples, 20 nmol of either 2'3'-cGAMP (Invivogen) or 3'2'-cGAMP (BioLog) were added to lysates from GFP transfected cells. The lysates were frozen in liquid nitrogen and stored at $-80^{\circ} \mathrm{C}$ for further analysis. $20 \mathrm{nmol}$ ATP$\gamma$-S (Jena Bioscience NU-506-5) were added to all lysates and nucleotides were purified from the lysates as previously described ${ }^{38}$ and then analyzed by means of LC-MS/MS with a 5500 QTrap (AB Sciex, Darmstadt, Germany) and a ExionLC AD UPLC (AB Sciex). For chromatographic separation, a Xbridge Amid $3.5 \mu \mathrm{m}, 150 \times 2.1 \mathrm{~mm}$ column (Waters) was used with acetonitrile/water with $5 \mathrm{mM}$ ammonium acetate $(50 / 50, \mathrm{v} / \mathrm{v}, \mathrm{pH} 9.5)$ as solvent $\mathrm{A}$ and acetonitrile/water with $5 \mathrm{mM}$ ammonium acetate $(95 / 5, \mathrm{v} / \mathrm{v}, \mathrm{pH} 9.5)$ as solvent $\mathrm{B}$. A 
linear gradient from $97 \%$ B to 50\% B in 16 min was used. Afterward the column was flushed and equilibrated to starting conditions. The separation of 2'3'-cGAMP, 3'2'-cGAMP and ATP- $\gamma-\mathrm{S}$ was performed using a $400 \mu \mathrm{L} \mathrm{m^{-1 }}$ flowrate at $40^{\circ} \mathrm{C}$ column oven temperature. Ions were analyzed by mass spectrometry in the negative ionization mode. The spray voltage was set to $-4,500 \mathrm{~V}$ at a source temperature of $450^{\circ} \mathrm{C}$ using nitrogen as collision gas. The parameters for the collision-activated dissociation (CAD) were: medium, curtain gas: 35 psi, ion source gas 1: 55 psi, ion source gas 2: 65 psi, entrance potential (EP) -10 V and the dwell time $150 \mathrm{msec}$. The MRM (multiple reaction monitoring) transition for each compound were as follow: cGAMP: $672.987 \rightarrow 79.1$ (quantifier), declustering potential (DP) $-205 \mathrm{~V}$, collision energy (CE) -160 V, Cell Exit Potential (CXP) -5 V; $672.987 \rightarrow 150.0$ (qualifier), DP -205V, CE -52 V, CXP -7 V; ATP- $\gamma$-S: $521.70 \rightarrow 78.8$ (quantifier), DP -115 V, CE -128 $\mathrm{V}, \mathrm{CXP}-11 \mathrm{~V} ; 521.70 \rightarrow 426.0$ (qualifier), DP $-115 \mathrm{~V}, \mathrm{CE}-32 \mathrm{~V}, \mathrm{CXP}-17 \mathrm{~V}$. Analyst 1.7. was used to acquire the data and MultiQuant 3.0.3 was used to analyze the data (both $A B$ Sciex).

\section{Expression and purification of recombinant protein}

E. coli BL21 (DE3) cells transformed with a pET-9d plasmid encoding cGLR1 were grown at $37^{\circ} \mathrm{C}$ in lysogenic broth medium containing $30 \mu \mathrm{g} / \mathrm{ml}$ kanamycin until the culture reached an $\mathrm{OD}_{600}$ of $0.4-0.5$. Protein expression was then induced with $1 \mathrm{mM}$ isopropyl-b-dthiogalactopyranoside and the cells were incubated for another $12 \mathrm{~h}$ at $16^{\circ} \mathrm{C}$. Afterwards, the cells were harvested by centrifugation, resuspended in Lysis Buffer $\left(50 \mathrm{mM} \mathrm{NaH} \mathrm{PO}_{4}\right.$, $500 \mathrm{mM} \mathrm{NaCl}, 10 \%$ glycerol, $5 \mathrm{mM} \beta$-mercaptoethanol, $1 \mathrm{mM} \mathrm{PMSF}, 200 \mu \mathrm{g} \mathrm{ml}^{-1}$ lysozyme, $\mathrm{pH}$ 7.4) and lysed by sonication. Following lysis, cell debris was removed by centrifugation at $18,500 \mathrm{rcf}$ for $60 \mathrm{~min}$ after which the supernatant was incubated with HisPur Ni-NTA Resin (ThermoFisher Scientific) at $4{ }^{\circ} \mathrm{C}$ for $1 \mathrm{~h}$ while continuously shaking. The resin was then transferred to a gravity flow column and washed with 20 column volumes $(\mathrm{CV})$ of Wash Buffer (50 mM NaH $\mathrm{PO}_{4}, 200 \mathrm{mM} \mathrm{NaCl}, 2 \mathrm{M}$ urea, 10\% glycerol, $5 \mathrm{mM} \beta$-mercaptoethanol, $\mathrm{pH}$ 7.4) before eluting the protein with $5 \mathrm{CV}$ of Elution Buffer (25 mM HEPES, $500 \mathrm{mM}$ $\mathrm{NaCl}, 500 \mathrm{mM}$ imidazole, $10 \%$ glycerol, $5 \mathrm{mM} \beta$-mercaptoethanol, $\mathrm{pH}$ 7.4). ). The eluate was diluted 2.5 times with Dilution Buffer (25 mM HEPES, $200 \mathrm{mM} \mathrm{KCl,} 5 \%$ glycerol, 5 $\mathrm{mM}$ dithiothreitol (DTT), $\mathrm{pH}$ 7.4) and then applied to a MBPTrap $1 \mathrm{ml}$ column (Cytiva). Bound protein was eluted with 5 CV Elution Buffer (20 mM HEPES, $200 \mathrm{mM} \mathrm{KCl,} \mathrm{10 \%}$ glycerol, $10 \mathrm{mM}$ maltose, $\mathrm{pH}$ 7.4) and TEV protease (one twentieth of the total protein concentration) was added before dialysis overnight at $4^{\circ} \mathrm{C}$ against Dialysis Buffer $(25 \mathrm{mM}$ 
Tris, $200 \mathrm{mM} \mathrm{KCl}, 5 \%$ glycerol, $5 \mathrm{mM}$ DTT, pH 7.4). Finally, the protein was aliquoted and flash frozen in liquid nitrogen before being stored at $-80^{\circ} \mathrm{C}$.

\section{In vitro activity assay}

cGLR1 activity was measured in a $50 \mu 1$ reaction volume containing $100 \mu \mathrm{g} / \mathrm{ml}$ recombinant cGLR1, $240 \mu \mathrm{M}$ ATP, $240 \mu \mathrm{M}$ GTP, $1 \mathrm{mM} \mathrm{MnCl}_{2}, 1 \mathrm{mM} \mathrm{MgCl}_{2}, 120 \mathrm{mM} \mathrm{KCl}$ and $2.5 \mathrm{ug}$ poly(I:C) (GE Healthcare), dsRNA (100 bp) or dsDNA (100 bp). The dsRNA was made as previously described ${ }^{39}$ whereas the dsDNA was made by purchasing complementary sense and antisense ssDNAs (Sigma-Aldrich) with sequences corresponding to the dsRNA and then annealing them. The reactions were incubated at $27^{\circ} \mathrm{C}$ for $4 \mathrm{~h}$ and then terminated at $95^{\circ} \mathrm{C}$ for $15 \mathrm{~min}$. To digest non-cyclic nucleotides, the reactions were afterwards incubated with $10 \mathrm{U}$ alkaline phosphatase (Roche) at $37^{\circ} \mathrm{C}$ overnight and again terminated at $95^{\circ} \mathrm{C}$ for $15 \mathrm{~min}$. The terminated reactions were applied to anion exchange chromatography as previously described $^{40}$ and 3',2'-cGAMP was quantified by integrating the corresponding peaks on the chromatogram using the software Unicorn 5.10 (GE Healthcare) with default settings.

\section{Treatment with CDNs}

12-well tissue culture plates were seeded with $3 \times 10^{5}$ HT-1080 or $3 \times 10^{6} \mathrm{~S} 2$ cells per well. After $20 \mathrm{~h}$, the medium was removed and $800 \mu \mathrm{l}$ digitonin buffer $(100 \mathrm{mM} \mathrm{KCl}, 85 \mathrm{mM}$ sucrose, $50 \mathrm{mM}$ HEPES, $3 \mathrm{mM} \mathrm{MgCl} 2,1 \mathrm{mM}$ ATP, $0.1 \mathrm{mM}$ GTP, $0.1 \mathrm{mM}$ DTT, $0.2 \%$ bovine serum albumin, $\mathrm{pH}$ 7.0) with or without $5 \mu \mathrm{M}$ 2',3'-cGAMP or 3',2'-cGAMP (Biolog Life Science Institute) was added to the wells. The cells were incubated for $10 \mathrm{~min}$ before the digitonin buffer was removed and replaced with normal medium.

\section{Ectopic expression of cGLRs in transgenic flies}

cGLRs were expressed in transgenic flies using the heat-controlled Gal4/Gal80 system. Briefly, the UAS-Gal4>cGLR transgenic flies were crossed with the driver line [actin5CGal4/CyO; tubulin-Gal80 $\left.{ }^{\mathrm{ts}} / \mathrm{TM} 6, \mathrm{~Tb}\right]$ at $25^{\circ} \mathrm{C}$. Males from $\mathrm{F} 1$ were collected and shifted to $29^{\circ} \mathrm{C}$ for 5 days to block the repressive function of Gal80 to Gal4 and drive the expression of the transgenes.

\section{Viral infections}

Viral stocks were prepared in $10 \mathrm{mM}$ Tris- $\mathrm{HCl}, \mathrm{pH}$ 7.5. Infections were performed with adult flies by intrathoracic injection (Nanoject II apparatus, Drummond Scientific) with $4.6 \mathrm{~nL}$ of 
DCV (5 (survival) or 500 (gene induction and viral load) PFU/fly), KV (10,000 ID $50 / f l y)$,

451

\section{RNA extraction from cells and flies and quantification of gene expression by qPCR}

RNA was extracted, cDNA was synthesized, and gene expression was quantified by qPCR as previously described ${ }^{21,37}$. Gene expression was analysed using the CFX Maestro Software (Bio-Rad).

\section{Statistical analyses}

All statistical analyses were done using GraphPad Prism 9.0.1 (GraphPad Software). Comparisons between groups were analysed using two-way ANOVA and Holm Holm-Š́íák post hoc test, except for comparison of viral RNA load and survival of flies. For comparison of viral load, data were log transformed and then analyzed using one-way ANOVA and Dunnett T3 post hoc test whereas survival of flies was compared using log-rank test. Sample sizes (n) for each experiment are stated in the figure legends.

\section{Data Availability Statement}

The authors declare that the data supporting the findings of this study are available within the paper. The sequences and structures used in this study are CG12970 (UniProt: A1ZA55), CG30424 (UniProt: A8DYP7), CG4746 (UniProt: Q9U3W6), CG4766 (UniProt: Q9Y106), CG7194 (UniProt: Q9VSH0), porcine OAS1 (UniProt: Q29599), human OAS1 (UniProt: P00973-1), human cGAS (UniProt: Q8N884-1), porcine cGAS (UniProt: I3LM39), murine cGAS (UniProt: Q8BSY1), and murine cGAS in complex with DNA and a cGAMP intermediate analog (PDB: 4K98). 

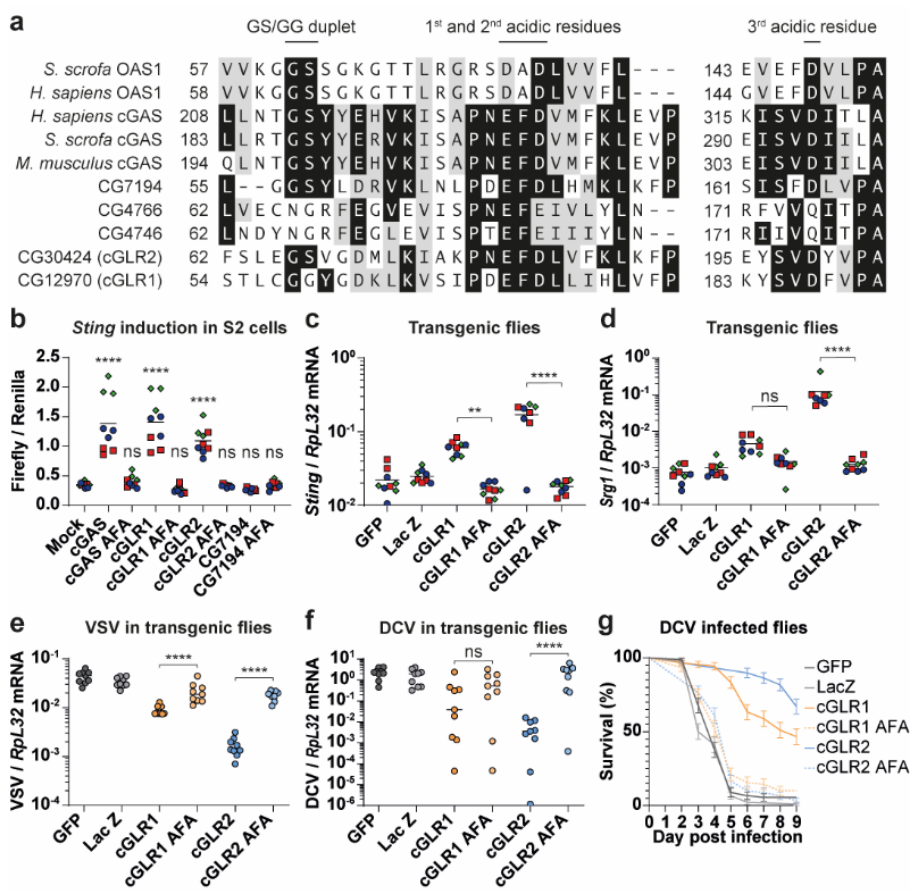

Fig. 1 | cGLR1 and cGLR2 activate the Sting pathway and protect against viral infection. a, Truncated alignment of identified cGAS-like candidates, mammalian cGAS and the structurally related OAS1. Essential active site residues (the GS/GG duplet and the metal ion coordinating acidic residues) are indicated above the alignment. b, Sting reporter activity in S2 cells transfected with cGAS-like candidates or their corresponding active site mutants (AFA). c, d, Expression of Sting (c) and Srgl (d) was monitored by RT-qPCR in transgenic flies ectopically expressing wild-type or mutant cGLRs. In b, c, d, data are from three independent experiments (blue, red, and green, each performed in triplicate) and shown with mean $(\mathrm{n}=9)$. e, f, VSV (e) or DCV (f) viral RNA load measured at 4 or 3 days, respectively, post infection in transgenic flies ectopically expressing wild-type or mutant cGLRs. Data are from three independent experiments, each performed in triplicates, and shown with mean $(\mathrm{n}=$ 9). g, Flies expressing the indicated transgenes were injected with DCV and survival was monitored daily. Data are from 3 independent experiments, each with 30 flies $(n=90)$. In $\mathbf{b}$, c, and d, data were analyzed using two-way ANOVA and Holm-Š́ídák post hoc test and compared to mock (a) or relevant AFA mutants $(\mathbf{b}, \mathbf{c}, \mathbf{d})$. In $\mathbf{e}$, and $\mathbf{f}, \log$ transformed data were analyzed using one-way ANOVA and Dunnett T3 post hoc test and compared to relevant AFA mutants. In g, log-rank test was used to compare cGLR1 vs. cGLR1 AFA $(P<$ $0.0001)$ and cGLR2 vs. cGLR2 AFA $(P<0.0001)$. ns not significant, $* * P<0.01$, **** $P<$ 0.0001 . 

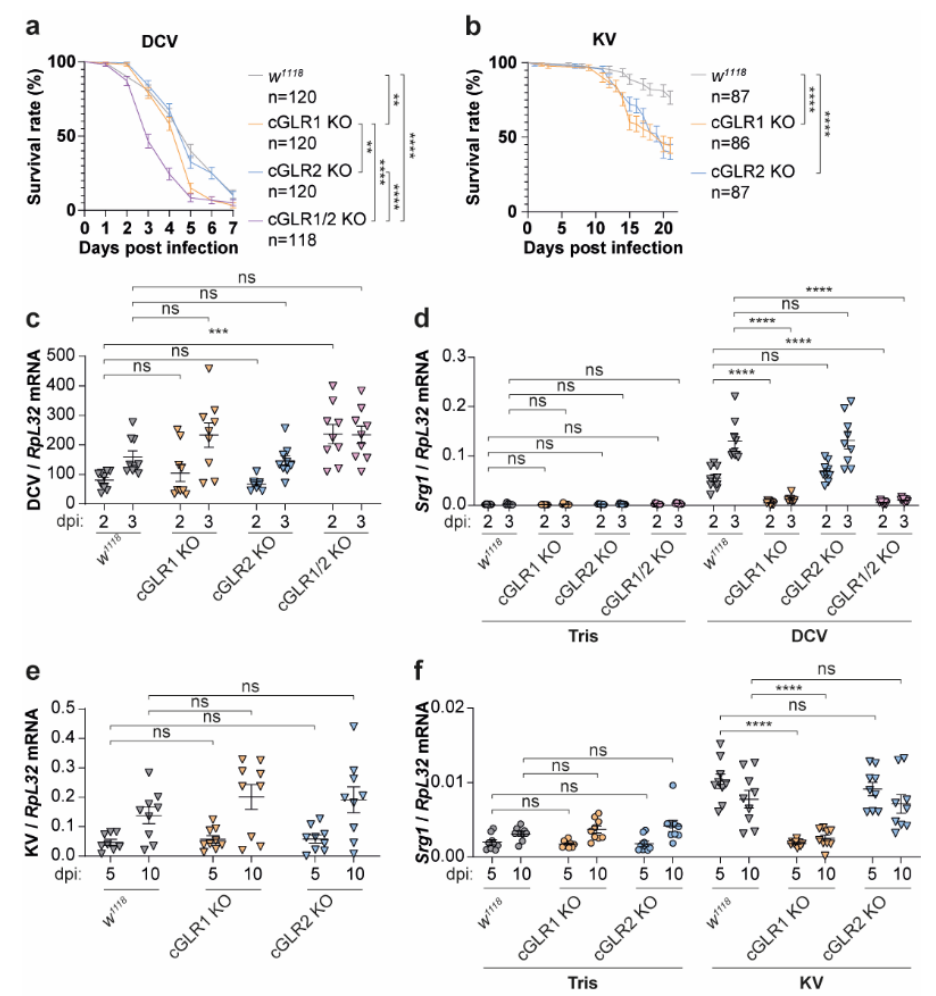

Fig. 2 | Loss of cGLR1 or cGLR2 leads to an impaired antiviral immune response in vivo. a, b, $w^{1118}$, cGLR1 KO, cGLR2 $\mathrm{KO}$ or cGLR1/2 KO flies were injected with DCV and survival was monitored daily. Data are from four independent experiments, each with three independent groups of around 10 flies. b, $w^{1118}$, cGLR1 KO or cGLR2 KO flies were injected with KV and survival was monitored daily. Data are from three independent experiments, each with three independent groups of around 10 flies. c, d, $w^{1118}$, cGLR1 KO, cGLR2 KO or cGLR1/2 KO flies were injected with DCV or Tris and viral load (c) as well as expression of Srgl (d) were monitored by RT-qPCR at 2 and 3 days post-injection (dpi). e, f, $w^{1118}$, cGLR1 KO or cGLR2 KO flies were injected with KV or Tris and viral load (e) as well as expression of $\operatorname{Srg} 1$ (f) were monitored by RT-qPCR at 5 and 10 days post-infection (dpi). For panels c-f, expression was normalized to the housekeeping gene $R p L 32$ and data are from three independent experiments, each performed in triplicates $(n=9)$. In $\mathbf{a}$ and $\mathbf{b}$, log-rank test was used to compare the survival curves pairwise followed by a Holm-Š́ídák multiple comparison correction. In $\mathbf{c}$ and $\mathbf{e}, \log$ transformed data were analyzed using one-way ANOVA and Dunnett T3 post hoc test and compared to $w^{1118}$ flies. In $\mathbf{d}$ and f, data were analyzed using two-way ANOVA and Holm-Š́íák post hoc test and compared to $w^{1118}$ flies. ns not significant, $* * P<0.01$. *** $P<0.001$.**** $P<0.0001$. 


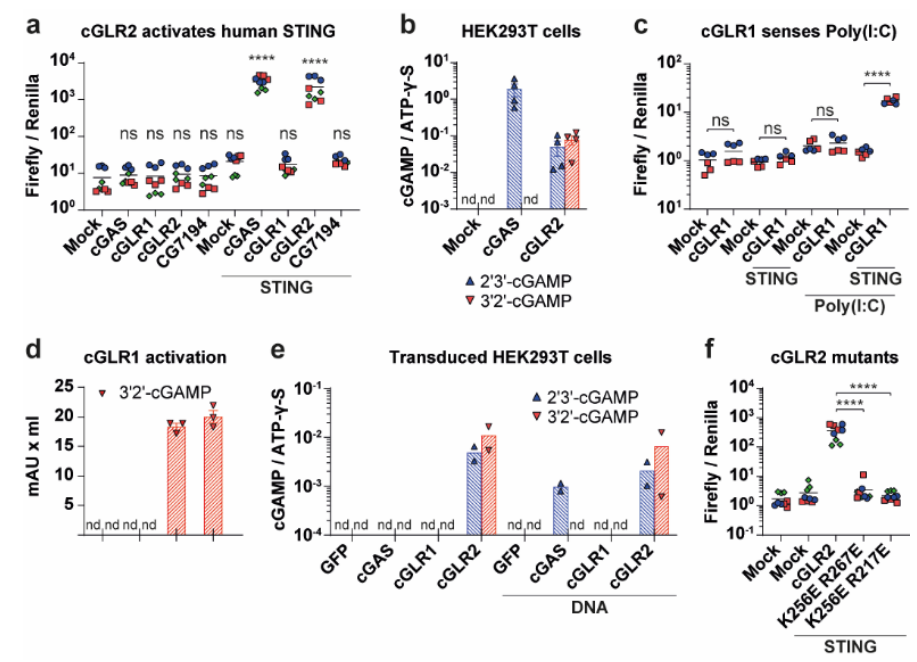
molecule. a, IFNB1 reporter activity in HEK293T cells transfected with cGAS-like candidates and human STING. Data are from three independent experiments (blue, red, and green, each performed in triplicates) and are shown with mean $(\mathrm{n}=9)$. b, Quantification by mass spectrometry of 2'3'-cGAMP or 3'2'-cGAMP production by HEK293T cells transfected with GFP, cGAS or cGLR2. Data are from four independent experiments and shown as mean \pm s.e.m. $(\mathrm{n}=4)$. c, IFNB1 reporter activity in HEK293T/MAVS KO cells transfected first with cGLR1 and human STING and afterwards with poly(I:C). Data are from two independent experiments (blue and red, each performed in triplicates) and are shown with mean $(n=9)$. d, Quantification by anion exchange chromatography analysis of 3'2'cGAMP production by recombinant cGLR1 in the presence of different nucleic acids. Data are from three independent experiments and shown as mean \pm s.e.m. $(n=3)$. e, Quantification by mass spectrometry of 2'3'-cGAMP or 3'2'-cGAMP production by HEK293T cells transduced with GFP, cGAS, cGLR1 or cGLR2 and subsequently transfected with DNA. Data are from two independent experiments $(\mathrm{n}=2)$. f, IFNB1 reporter activity in HEK293T cells transfected with wild-type cGLR2 or the indicated mutants and human STING. Data are from three independent experiments (blue, red, and green, each performed in triplicates) and are shown with mean $(\mathrm{n}=9)$. In $\mathbf{a}, \mathbf{c}$ and $\mathbf{f}$, data were analyzed using two-way ANOVA and 565 


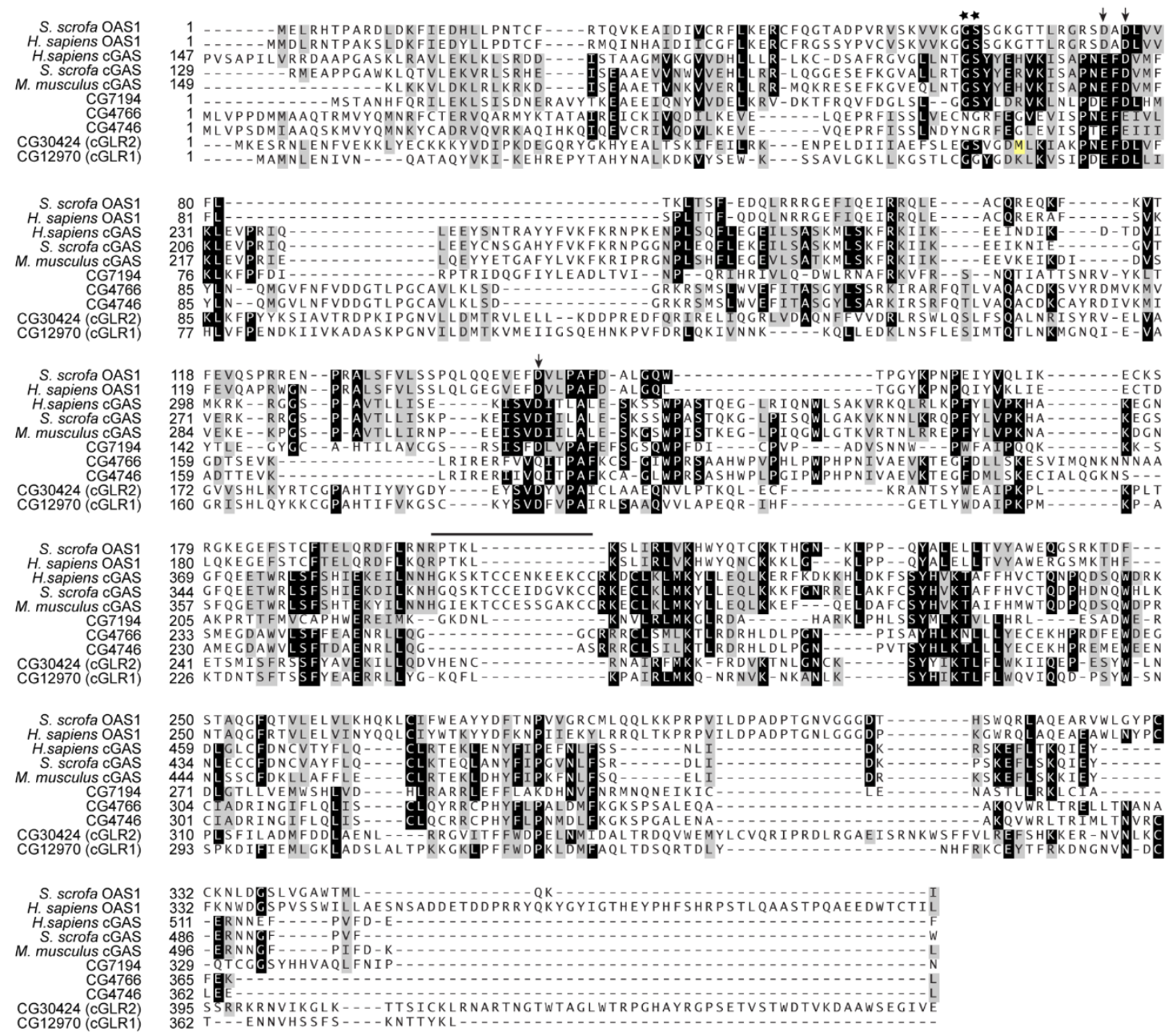

b

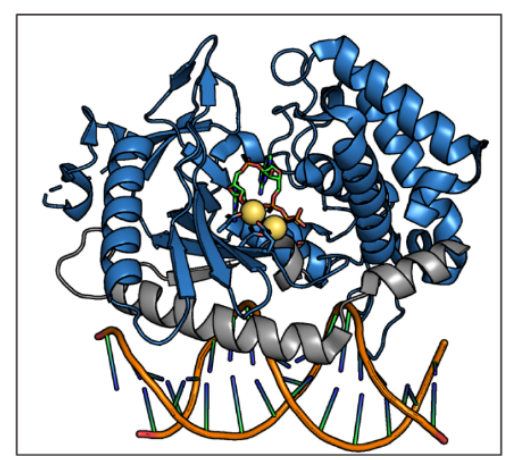

c

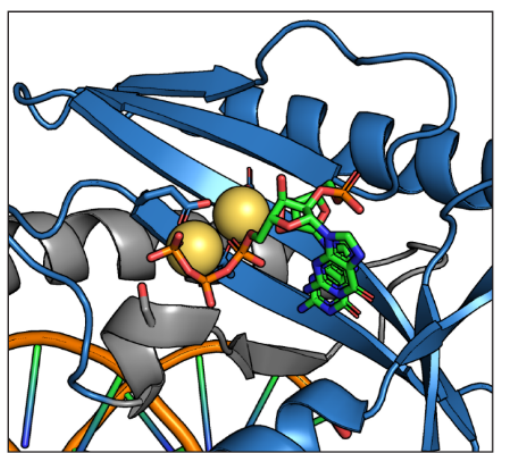

Extended Data Fig. 1 | Alignment of mammalian cGAS/OAS1 proteins and cGAS-like

proteins from D. melanogaster. a, Extended view of the alignment shown in Fig. 1a with the positions of the GS/GG duplet, the metal ion coordinating acidic residues, and the $\mathrm{Zn}$-finger indicated above the sequences with stars, arrows and bar, respectively. The annotated start methionine in the NCBI reference sequence for isoform D of CG30424 (cGLR2), which would delete the entire spine helix as well as part of the active site, is highlighted in yellow. 
573 Deletion of the spine helix is incompatible with a folded enzyme. Furthermore, the GS$574 \quad$ containing loop, which coordinates the $\gamma$-phosphate of the donor nucleotide, is a universally 575 conserved feature of nucleotidyltransferases. b, c, Structure (PDB: 4K98) of murine cGAS in 576 complex with DNA and a cGAMP intermediate analog (represented in sticks) and two 577 magnesium ions (yellow spheres). The sidechains of the acidic active site residues and the 578 serine in the GS motif are also represented in sticks and their oxygen atoms are colored red. 579 Grey coloring represents the proportion of the protein upstream of the annotated start 580 methionine site in CG30424 (cGLR2). b, Full view of the structure. c, Enhanced view of the active site. 
a b
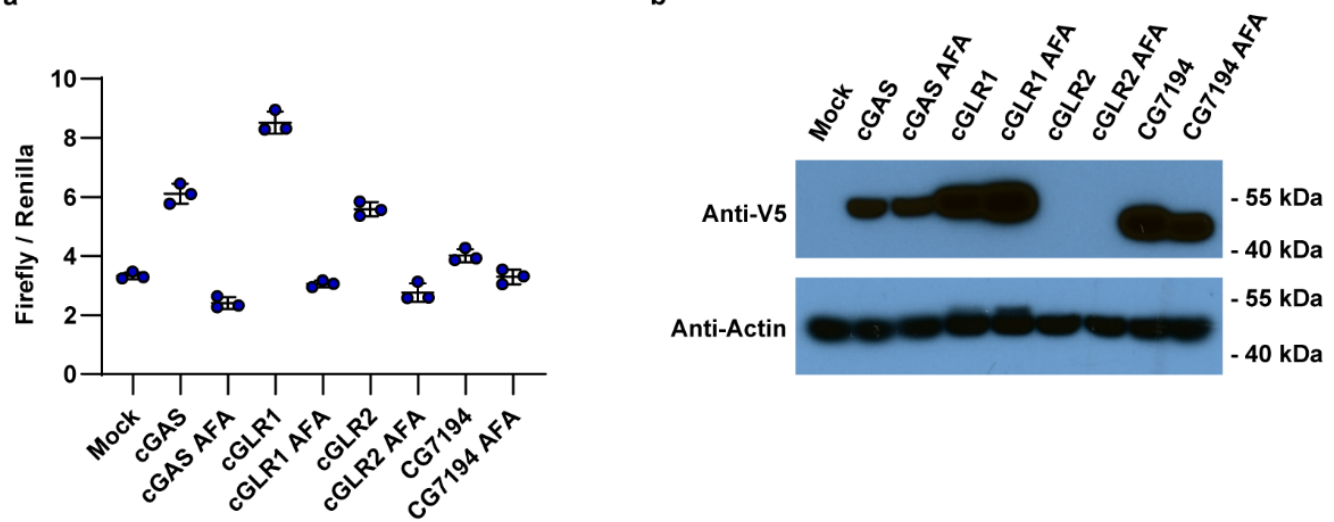

Extended Data Fig. 2 | Transient expression of candidate cGAS-like receptors in S2 cells. a, Due to issues with the ANTI-FLAG ${ }^{\circledR}$ M2 antibody giving rise to non-specific bands when performing immunoblots on S2 cell lysates, we replaced the FLAG-tag we initially used with a V5-tag and reproduced the experiment from Fig. 1b. Cells were transfected with cGAS, cGLR1, cGLR2 and CG7194 or mutants thereof as well as plasmids encoding firefly or Renilla luciferase under transcriptional control of the Sting or Actin5C promoter, respectively. At $24 \mathrm{~h}$ post transfection, luciferase activity was measured. Data are from one experiment performed in triplicates and are shown with mean \pm s.d. $(n=3) \mathbf{b}$, Immunoblot showing the expression of the V5-tagged proteins from panel (a). cGLR2 appears to be rapidly degraded in S2 cells, whereas it could easily be detected in HEK293T cells following transfection (Extended Data Fig. 10a). For gel source data, see Supplementary Fig. 1. 
a

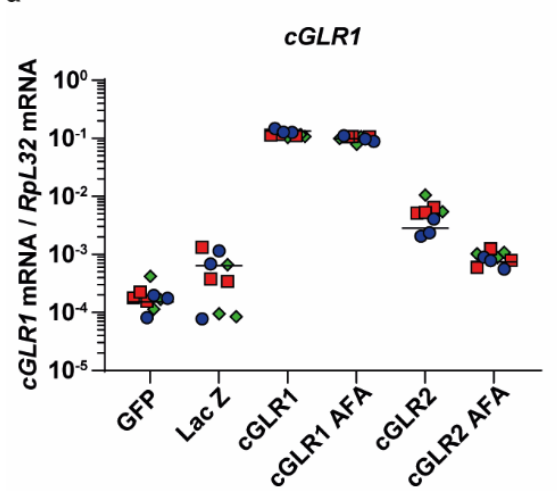

C

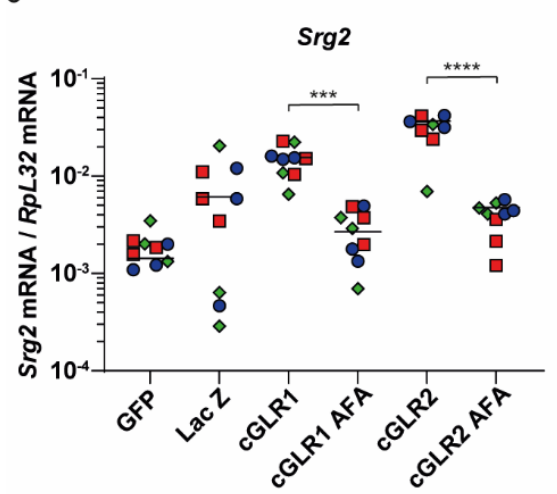

b

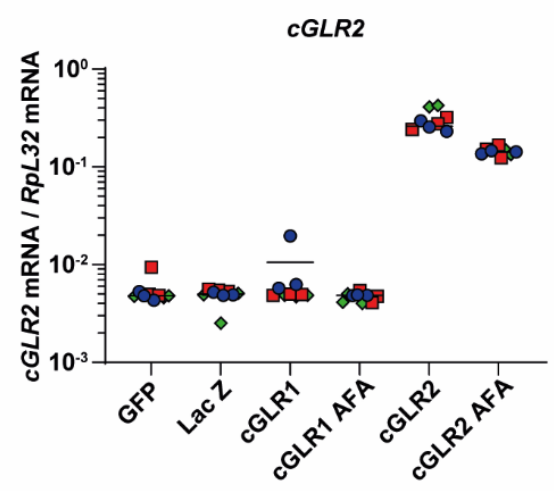

d

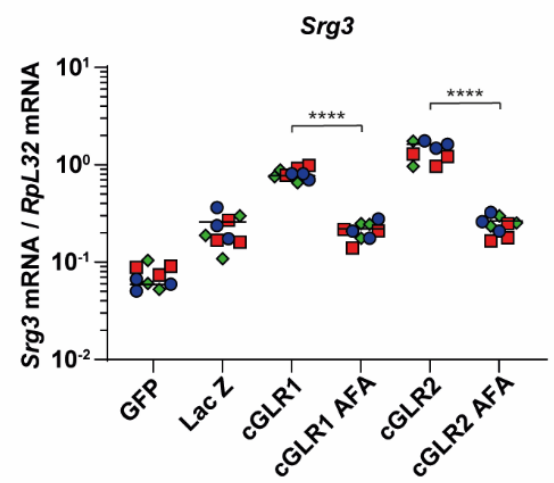

e

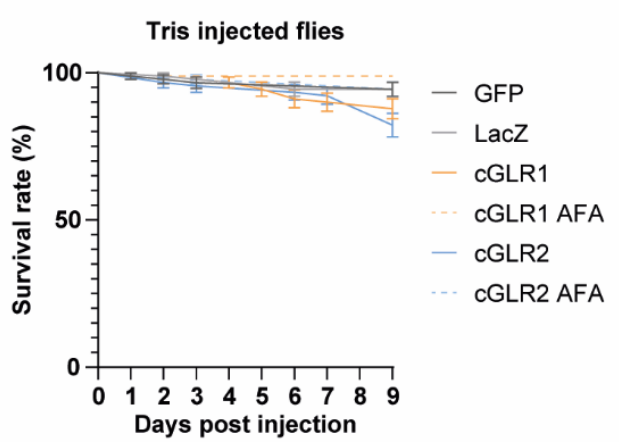

Extended Data Fig. 3 | Ectopic expression of cGLR1 or cGLR2 in transgenic flies induces expression of $\mathbf{s r g} 2$ and $\mathbf{s r g} 3$. a, b, c, d, Expression of $c G L R 1$ (a), cGLR2 (b), Srg2 (c) and $\operatorname{Srg} 3$ (d) was monitored by RT-qPCR in transgenic flies ectopically expressing wildtype or mutant cGLRs. Expression was normalized to the housekeeping gene RpL32. Data are from three independent experiments (red, blue and green, each performed in triplicate) and shown with mean $(n=9)$. e, Flies of the indicated genotypes were injected with Tris and survival was monitored daily. Data are from 3 independent experiments, each with 30 flies (n =90). In a, b, c, and d, data were analysed using two-way ANOVA and Holm-Š́dák post hoc test and compared to relevant AFA mutants. In e, log-rank test was used to compare cGLR1 

vs. cGLR1 AFA (ns) and cGLR2 vs. cGLR2 AFA (ns). ns not significant, ${ }^{*} \mathrm{P}<0.05$. $* * \mathrm{P}<$ $605 \quad 0.01 . * * * \mathrm{P}<0.001 . * * * * \mathrm{P}<0.0001$. 


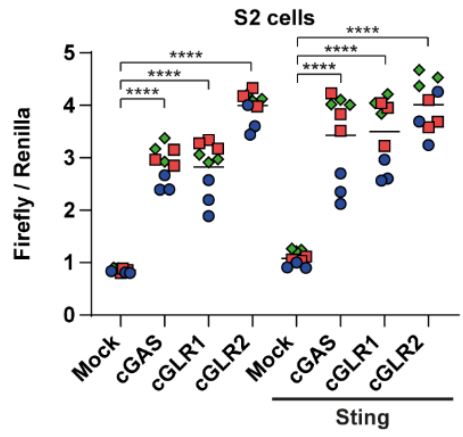

b

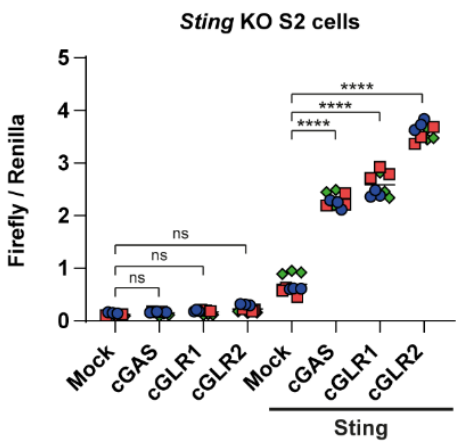

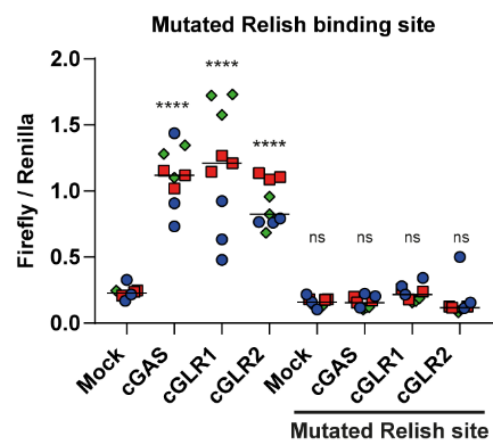

d

e
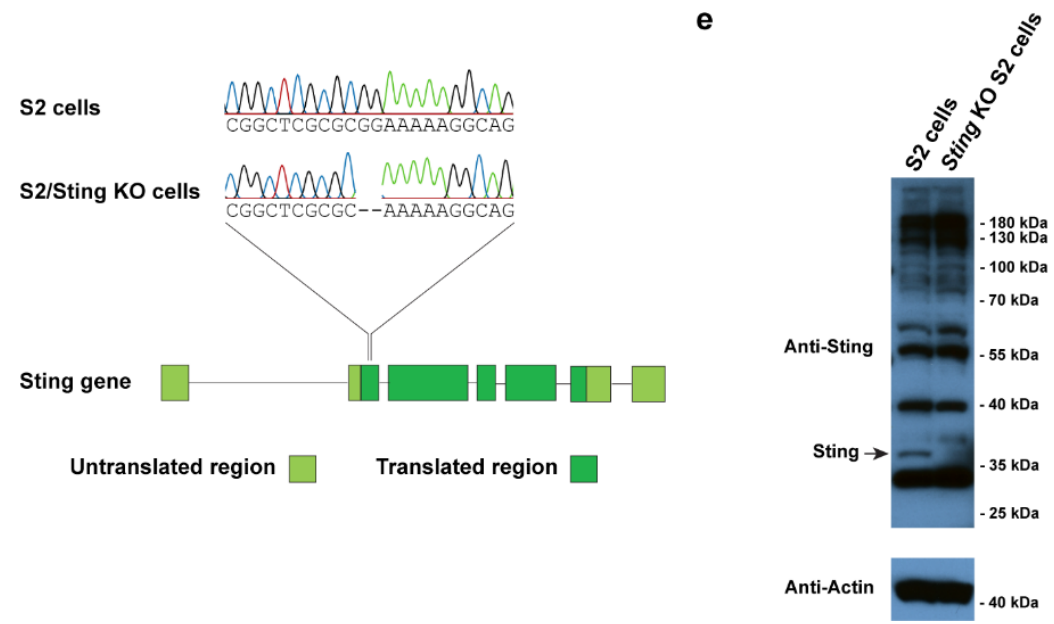

Extended Data Fig. 4 | Verification of Sting knockout S2 cells. S2 cells transfected with expression vectors for cGAS, cGLR1 or cGLR2 and plasmids encoding firefly or Renilla luciferase under transcriptional control of the Sting or Actin5C promoter, respectively, were used to monitor activation of the Sting pathway. a, S2 cells with or without co-transfection with an expression vector for Sting. b, Sting knockout (KO) S2 cells with or without cotransfection with an expression vector for Sting. c, S2 cells transfected with expression vectors for cGAS, cGLR1 or cGLR2 and plasmid encoding firefly luciferase under transcriptional control of the Sting promoter or a mutated version containing two point mutations in the Relish binding site. Data are from three independent experiments (blue, red, and green, each performed in triplicates) and are shown with mean $(\mathrm{n}=9)$. Data were analyzed using two-way ANOVA and Holm-Š́ídák post hoc test and compared to mock. ns not significant, $* * * * P<0.0001$. d, Sanger sequencing showing the 2 bp deletion in the Sting gene in Sting knockout (KO) S2 cells. e, Immunoblot showing the lack of expression of Sting in Sting KO S2 cells. The arrow indicates the position of the Sting band. For gel source data, see Supplementary Fig. 1. 

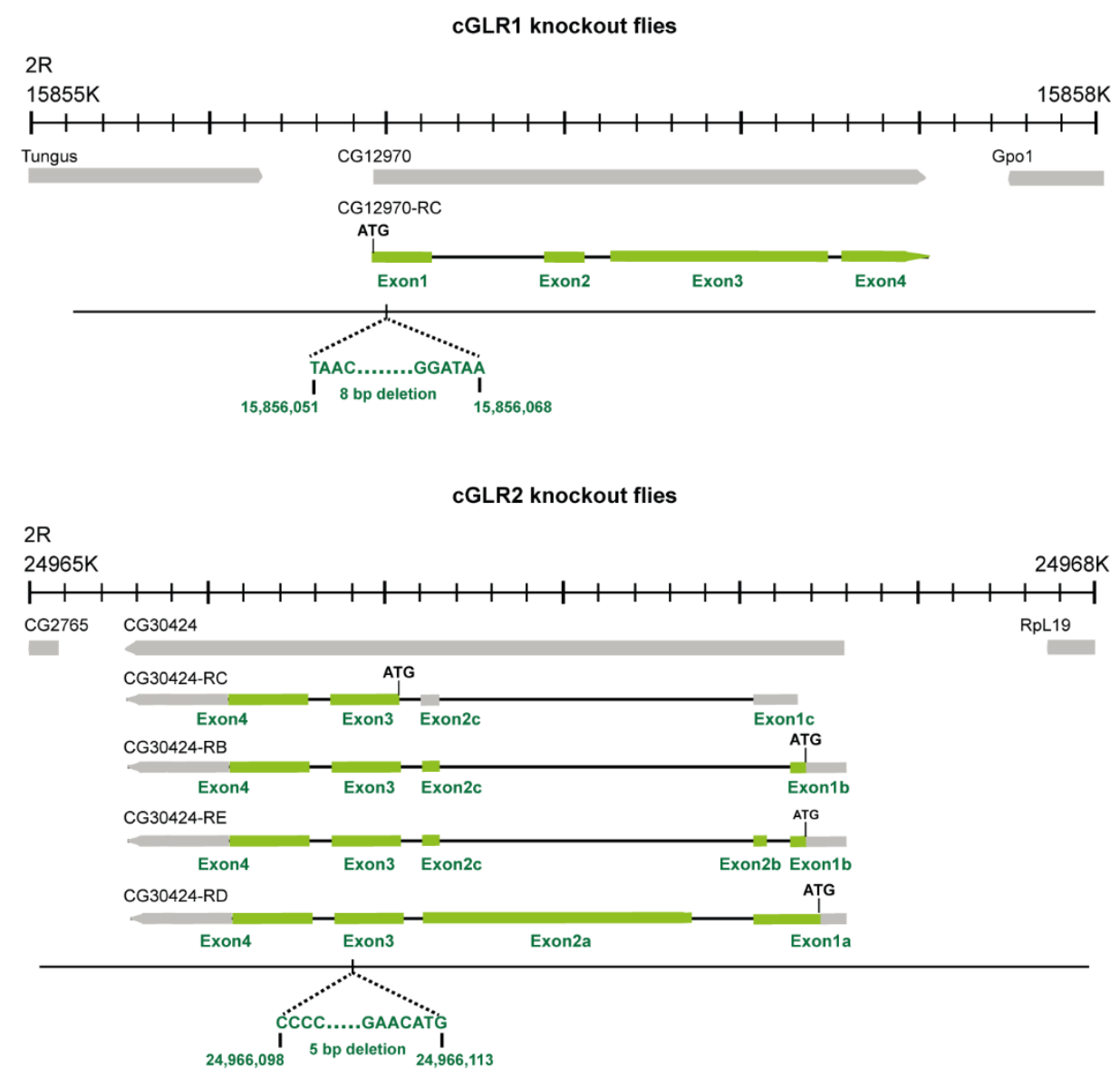

Extended Data Fig. 5 | Generation of $\boldsymbol{c} G L R 1$ and $\boldsymbol{c} G L R 2$ knockout flies. The $c G L R 1$ and $c G L R 2$ genes, both located on the right arm of the second chromosome, are shown together with their annotated transcripts. Open reading frames are indicated in green. For $c G L R 1$, an 8 bp deletion was introduced in the first exon using CRISPR/Cas9 technology. The deletion creates a frameshift after the asparagine residue at position 31, leading to termination of translation after insertion of single glycine residue. For $c G L R 2$, a 5 bp deletion was created in exon 3, which is shared by all isoforms. The deletion results in a frameshift after the glutamate residue at position 338, leading to termination of translation after insertion of a 32 amino acid extension (HDRRIDPGSSLGNVPVRAKDSKRPEGRRDQPE). 


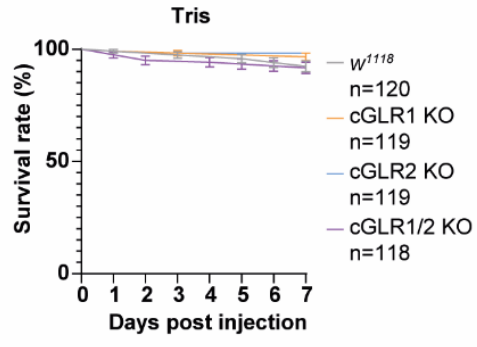

c

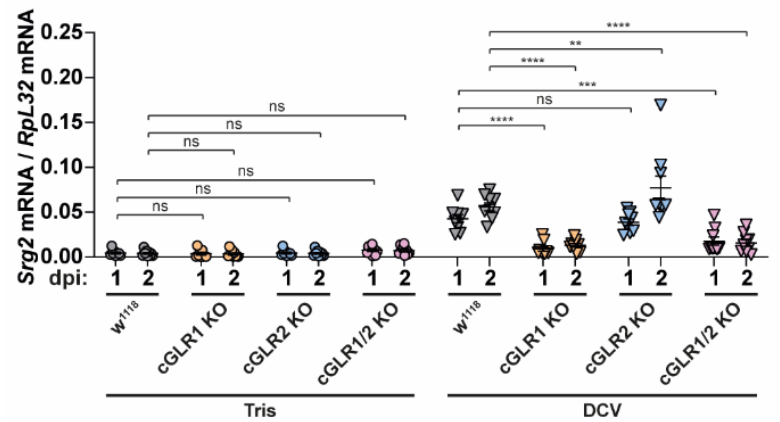
$P<0.001$.**** $P<0.0001$.
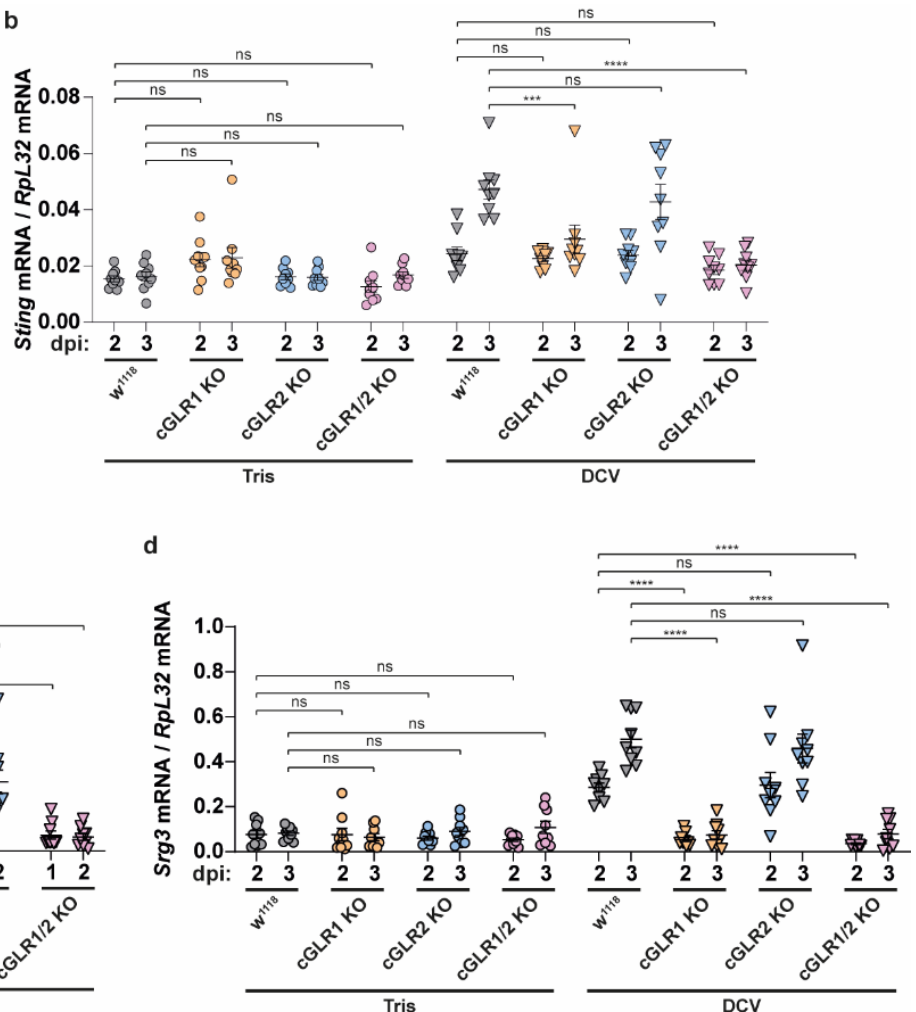
Extended Data Fig. 6 | Expression of Sting, Srg2 and Srg3 in flies upon infection with DCV. a, Corresponding control to Fig. 2a. $w^{1118}$, cGLR1 KO, cGLR2 KO or cGLR1/2 KO flies were injected with Tris and survival was monitored daily. Data are from three independent experiments, each with three groups of around 10 flies. b, c, d, $w^{111}$, cGLR1 $\mathrm{KO}$, cGLR2 KO or cGLR1/2 KO flies were injected with DCV or Tris and expression of Sting (a), Srg2 (b) and Srg3 (c) was monitored by RT-qPCR at 2 and 3 days post-infection (dpi). Expression was normalized to the housekeeping gene RpL32. Data are from three independent experiments, each performed in triplicates $(n=9)$. In a, log-rank test was used to test if the survival curves differed. In $\mathbf{b}, \mathbf{c}$ and $\mathbf{d}$, data were analyzed using two-way ANOVA and Holm-Š́́dák post hoc test and compared to $w^{1118}$ flies. ns not significant, ** $P<0.01$. *** 

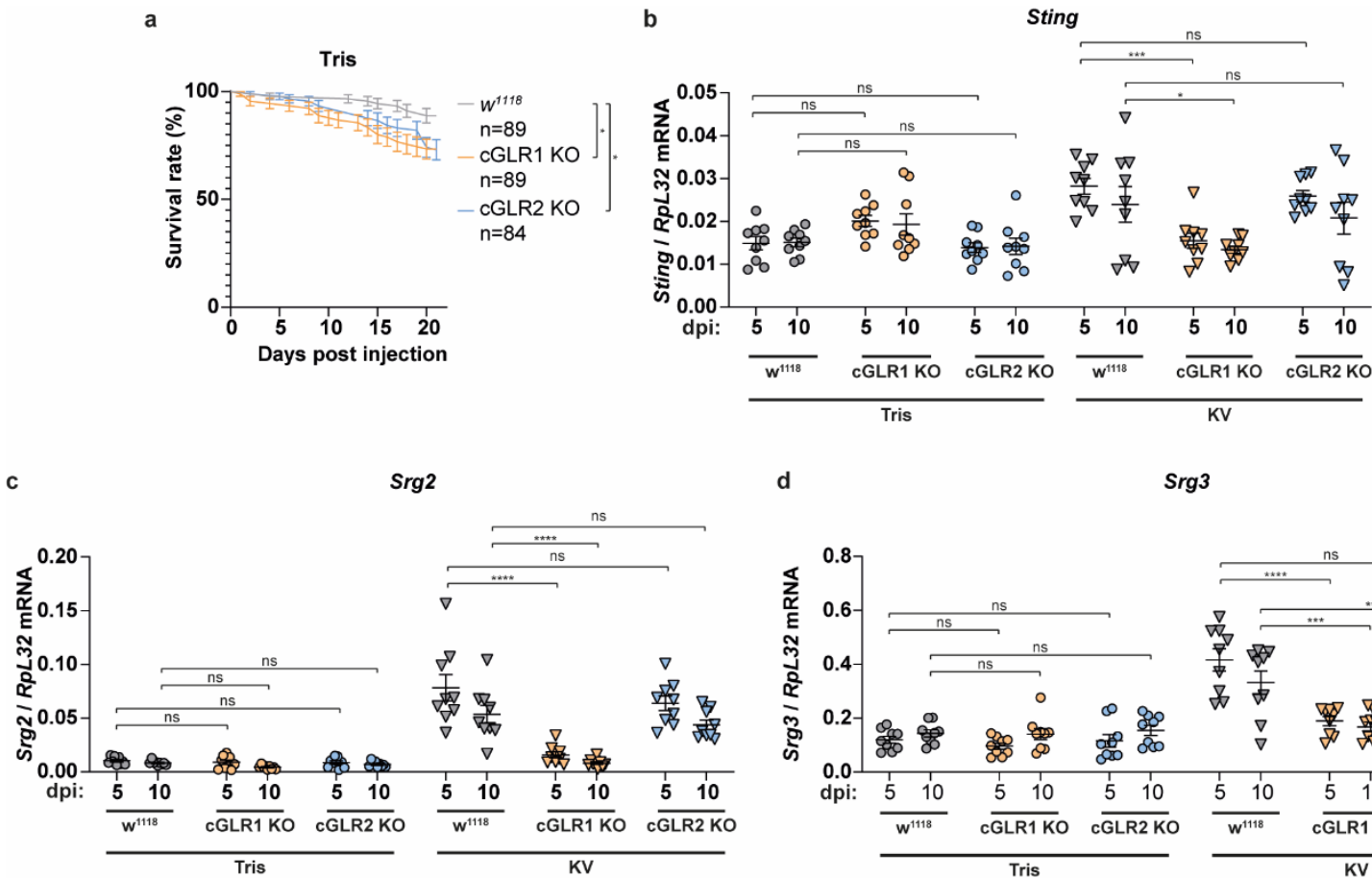

d $\quad S r g 3$

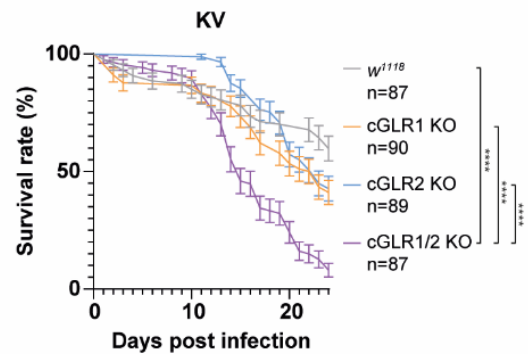

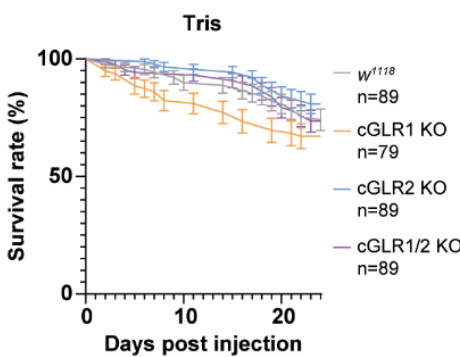

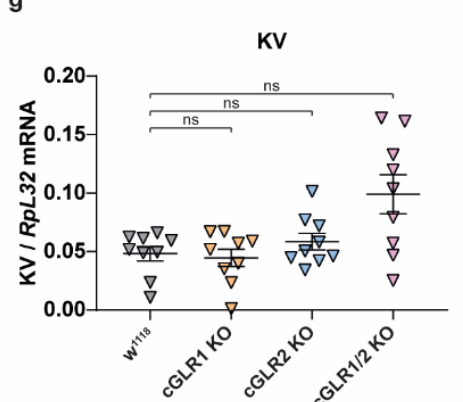

Extended Data Fig. 7 | Expression of Sting, Srg2 and Srg3 in flies upon infection with KV. a, Corresponding control to Fig. 2b. $w^{1118}$, cGLR1 KO or cGLR2 KO flies were injected with Tris and survival was monitored daily. Data are from three independent experiments, each with three groups of around 10 flies. b, c, d, $w^{1118}$, cGLR1 KO or cGLR2 KO flies were injected with KV or Tris and expression of Sting (a), Srg2 (b) and Srg3 (c) was monitored by RT-qPCR at 5 and 10 days post-infection (dpi). Expression was normalized to the housekeeping gene $R p L 32$. ). Data are from three independent experiments, each performed in triplicates $(\mathrm{n}=9)$. e, $\mathbf{f}, w^{1118}$, cGLR1 KO, cGLR2 KO or cGLR1/2 KO flies were injected with KV (e) or Tris (f) and survival was monitored daily. Data are from three independent experiments, each with three groups of around 10 flies. g, $w^{1118}$, cGLR1 KO, cGLR2 or cGLR1/2 KO flies were injected with $\mathrm{KV}$ and viral load was monitored by RT-qPCR at 5 days post-infection (dpi). Expression was normalized to the housekeeping gene RpL32. Data are from three independent experiments, each performed in triplicates $(n=9)$. In $\mathbf{a}, \mathbf{e}, \mathbf{f}, \log$ - 
Extended Data Fig 7 | Loss of cGLR1 or cGLR2 has a limited effect on VSV infection in flies
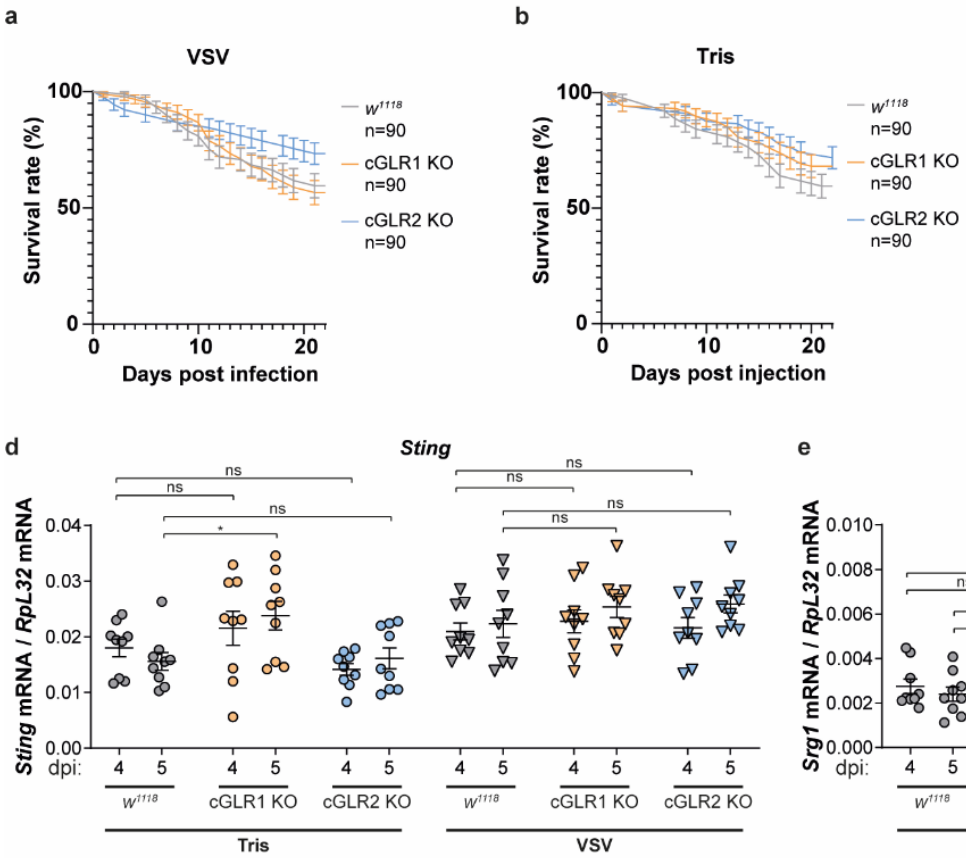

e



Srg1

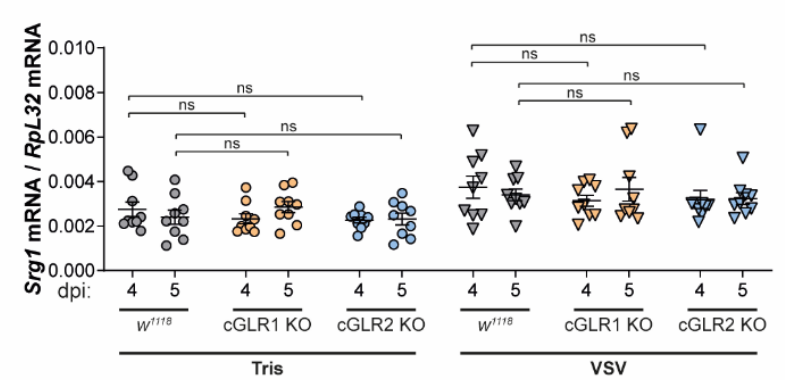

g
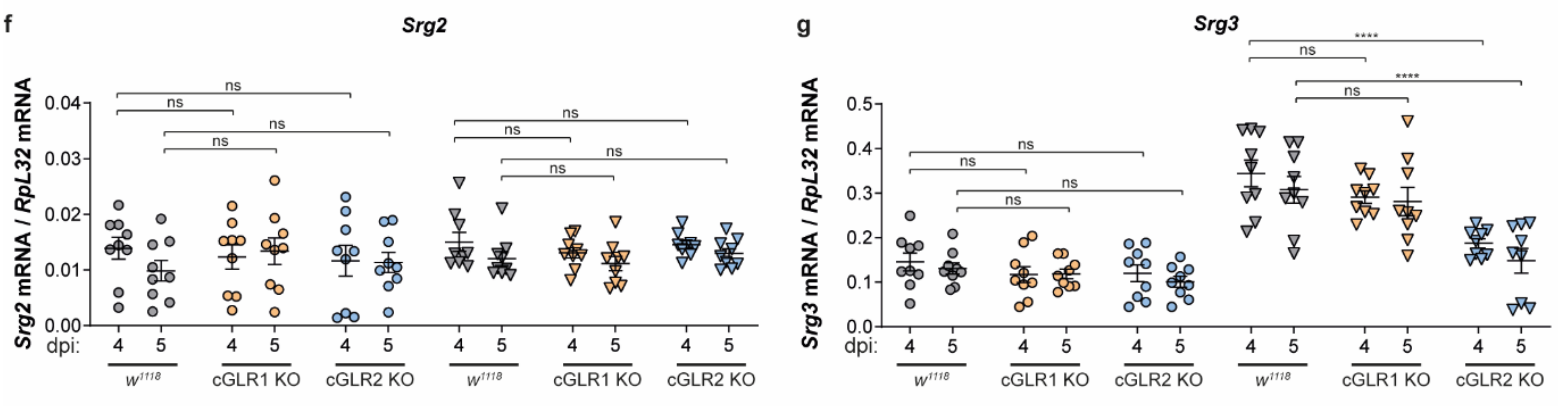

Tris

flies. a, b, $w^{1118}$, cGLR1 KO or cGLR2 KO flies were injected with VSV (a) or Tris (b) and 
survival was monitored daily. Data are from three independent experiments, each with three groups of around 10 flies. $\mathbf{c}, \mathbf{d}, \mathbf{e}, \mathbf{f}, \mathbf{g}, w^{1118}$, cGLR1 KO, cGLR2 KO or cGLR1/2 KO flies were injected with VSV or Tris and viral load (c) as well as expression of Sting (d), Srgl (e), Srg2 (f) and $\operatorname{Srg} 3$ (g) were monitored by RT-qPCR at 4 and 5 days post-infection (dpi). Expression was normalized to the housekeeping gene RpL32. Data are from three independent experiments, each performed in triplicates $(n=9)$. In $\mathbf{a}$ and $\mathbf{b}, \log$-rank test was used to test if the survival curves differed. In c, Log transformed data were analyzed using one-way ANOVA and Dunnett T3 post hoc test and compared to $w^{1118}$ flies. In d, e, f and $\mathbf{g}$, data were analyzed using two-way ANOVA and Holm-Š́íák post hoc test and compared to $w^{1118}$ flies. ns not significant, ${ }^{*} P<0.05$. *** $P<0.001{ }^{* * * *} P<0.0001$. 

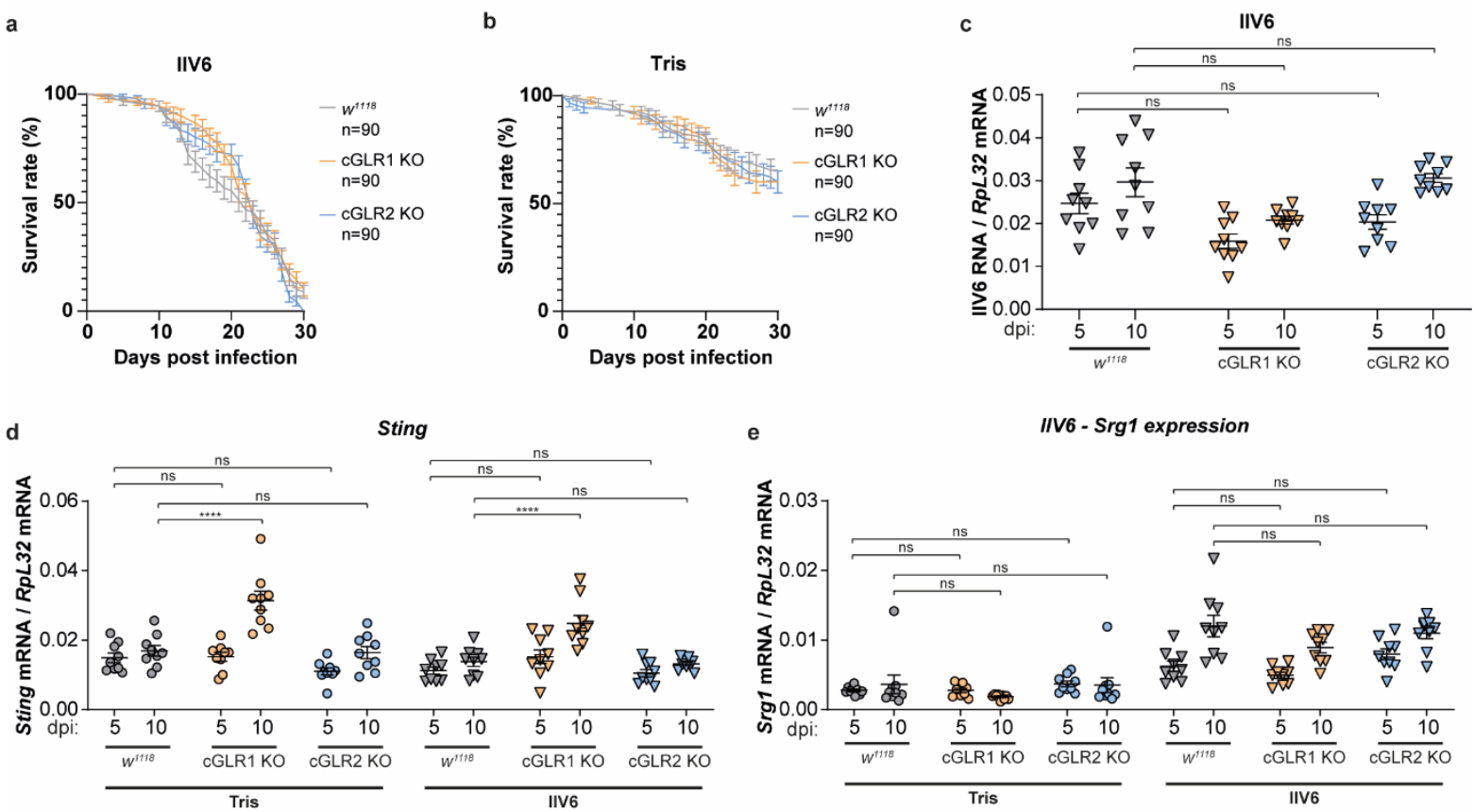

IIV6 - Srg1 expression
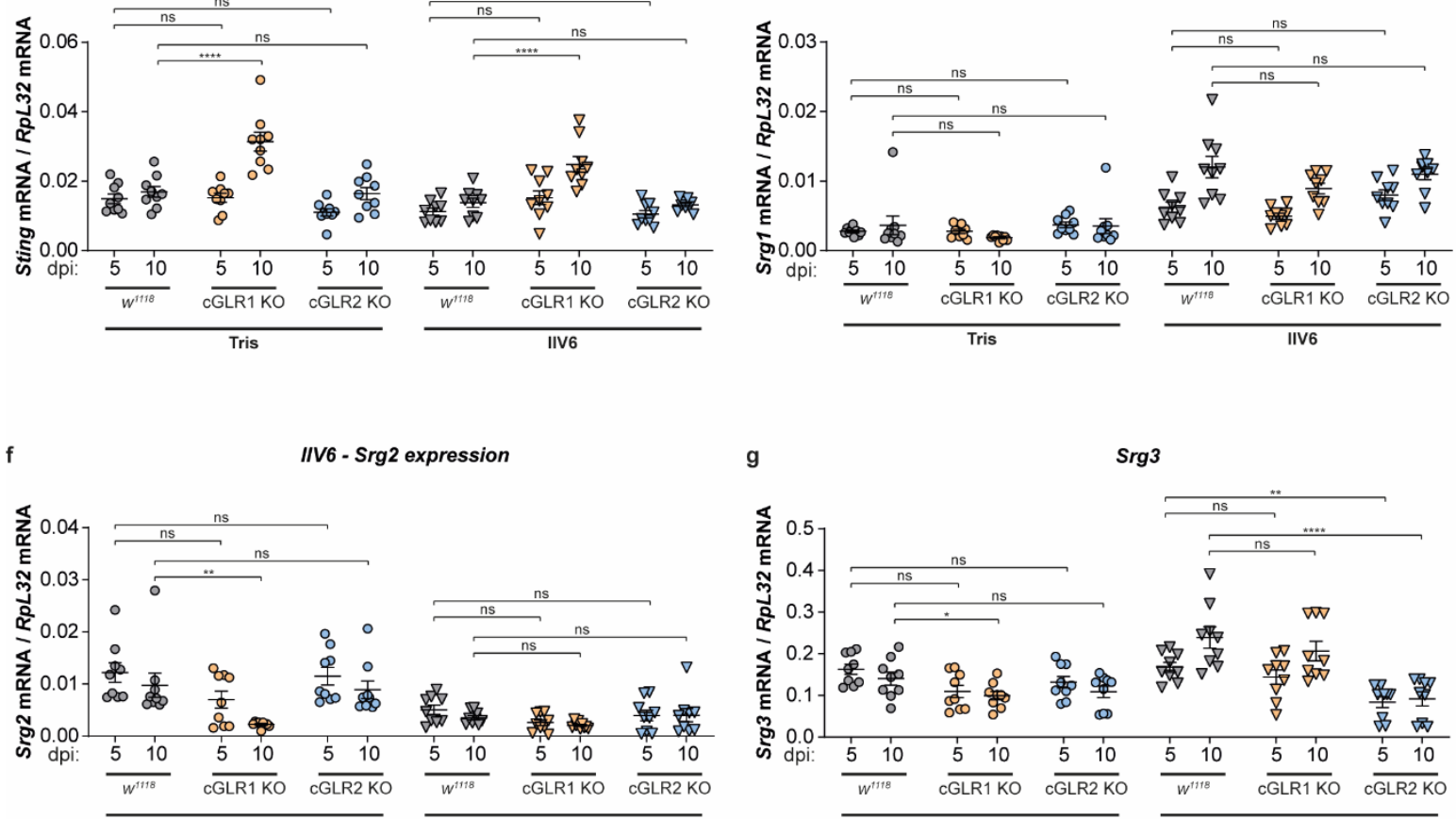

flies. a, b, $w^{1118}$, cGLR1 KO or cGLR2 KO flies were injected with IIV6 (a) or Tris (b) and survival was monitored daily. Data are from three independent experiments, each with three groups of around 10 flies. $\mathbf{c}, \mathbf{d}, \mathbf{e}, \mathbf{f}, \mathbf{g}, w^{1118}$, cGLR1 KO or cGLR2 KO flies were injected with IIV6 or Tris and viral load (c) as well as expression of Sting (d), Srg1 (e), Srg2 (f) and Srg3 (g) were monitored by RT-qPCR at 5 and 10 days post-infection (dpi). Expression was normalized to the housekeeping gene $R p L 32$. Data are from three independent experiments, each performed in triplicates $(n=9)$. In $\mathbf{a}$ and $\mathbf{b}$, log-rank test was used to test if the survival curves differed. In c, Log transformed data were analyzed using one-way ANOVA and Dunnett T3 post hoc test and compared to w1118 flies. In $\mathbf{d}, \mathbf{e}, \mathbf{f}$ and $\mathbf{g}$, data were analyzed using two-way ANOVA and Holm-Š́́dák post hoc test and compared to w1118 flies. ns not significant, ${ }^{*} P<0.05$. ${ }^{* *} P<0.01$. *** $P<0.001$. **** $P<0.0001$. 


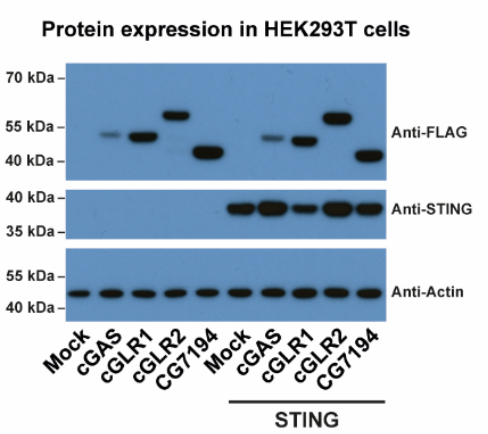

d
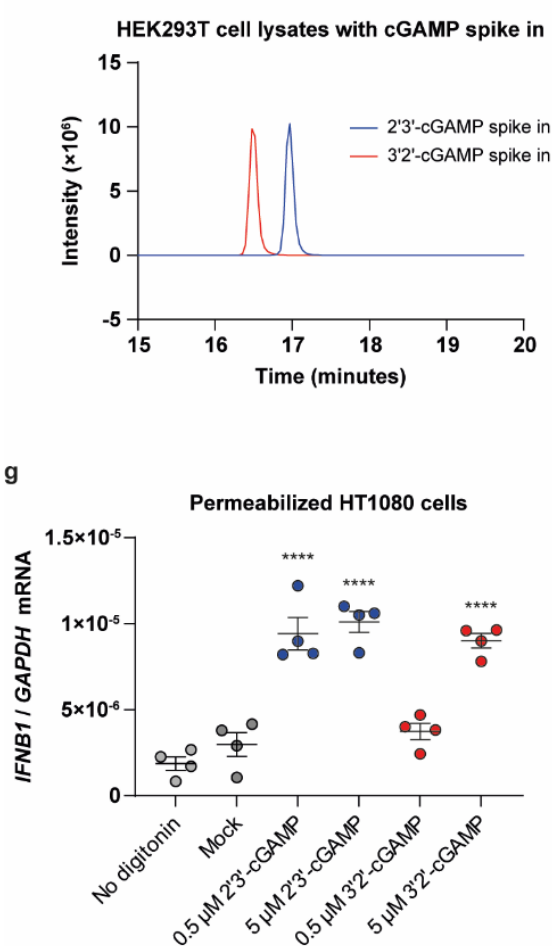

b
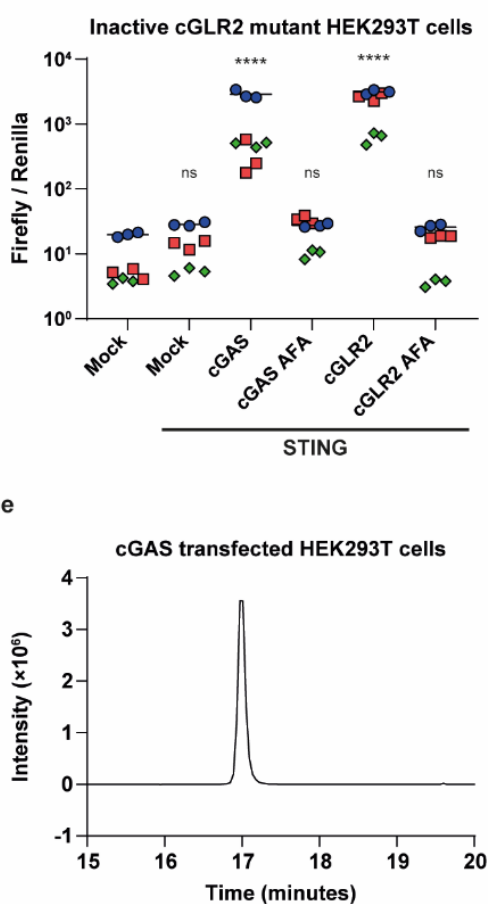

h

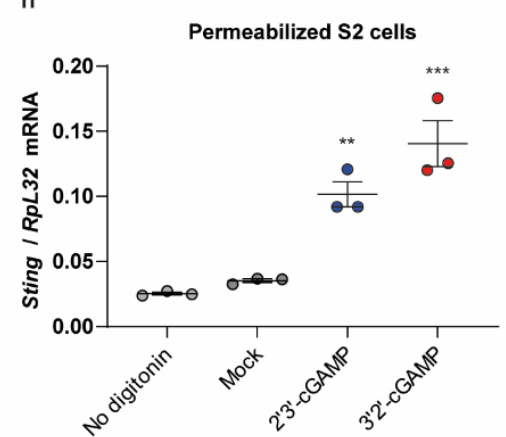


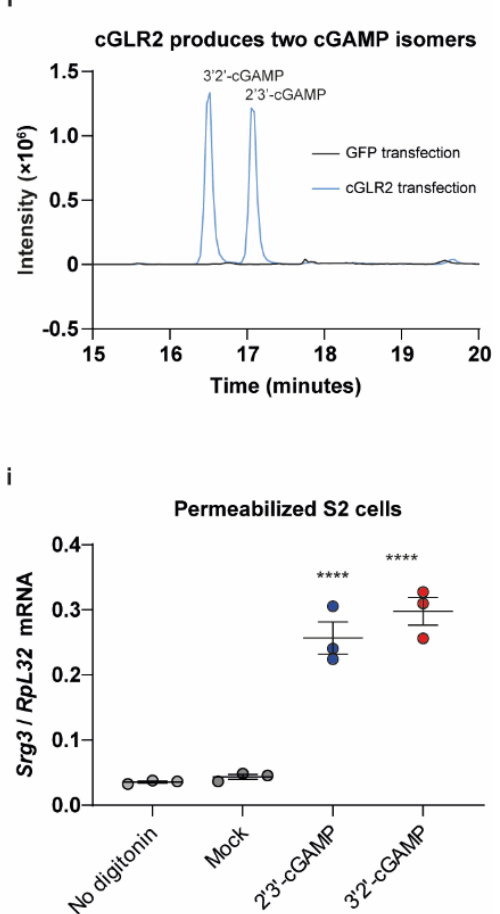

Extended Data Fig. 10 | cGLR2 produce 2'3'-cGAMP and 3'2'-cGAMP, which can activate human and Drosophila STING. a, Immunoblot showing the expression of STING and FLAG-tagged nucleotidyltransferases from Fig. 3a. b, HEK293T cells transfected with cGAS, cGAS AFA, cGLR2, cGLR2 AFA, and STING as indicated and plasmids encoding firefly or Renilla luciferase under transcriptional control of the IFNB1 or a constitutive promoter, respectively. Data are from three independent experiments (blue, red, and green, each performed in triplicates) and shown with mean $(n=9)$. c, Immunoblot showing the expression of STING and FLAG-tagged nucleotidyltransferases from panel (b). d, Representative chromatograms from mass spectrometry analysis of 2'3'-cGAMP or 3'2'cGAMP spiked lysates from GFP transfected cells. e, Representative chromatogram from mass spectrometry analysis of cGAS transfected cells. f, Representative chromatograms from mass spectrometry analysis of GFP or cGLR2 transfected cells. g, h, i, HT-1080 (g) or S2 
cells (h and i) permeabilized using digitonin and treated with the indicated cGAMPs. Expression of IFNB1 (g), Sting (h) or Srgl (i) were monitored by RT-qPCR at 6 and 8 h post treatment for S2 and HT-1080 cells, respectively. Expression was normalized to the housekeeping genes GAPDH (g) and $R p L 32$ (h and i). Data are shown with mean \pm s.e.m. (n $=4$ for $\mathbf{g}$ and $\mathbf{n}=3$ for $\mathbf{h}$ and $\mathbf{i}$ ). In $\mathbf{b}$, data were analyzed using two-way ANOVA and HolmŠ́lák and compared to mock. In $\mathbf{g}, \mathbf{h}$, $\mathbf{i}$, data were analyzed using one-way ANOVA and Dunnett T3 post hoc test and compared to mock. ns not significant, ${ }^{* *} P<0.01$. ${ }^{* * *} P<$ $0.001 . * * * * P<0.0001$. 


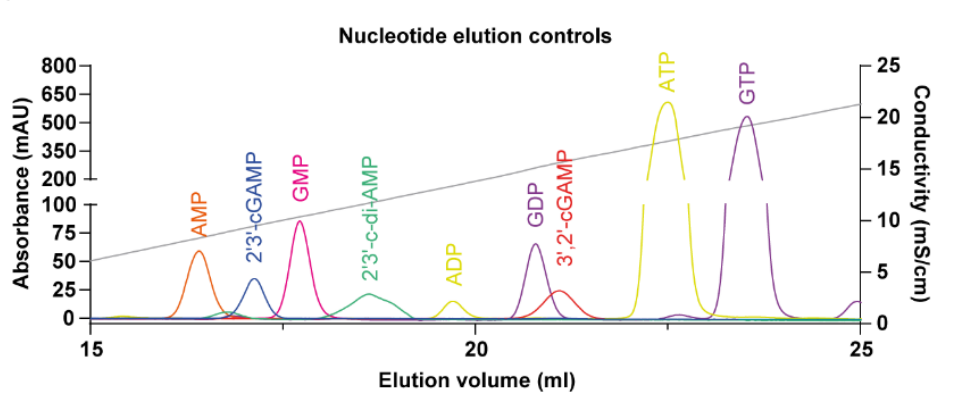

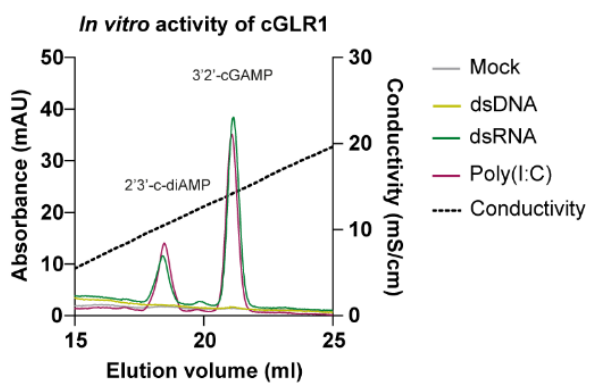

cGLR2 expression in HEK293T cells

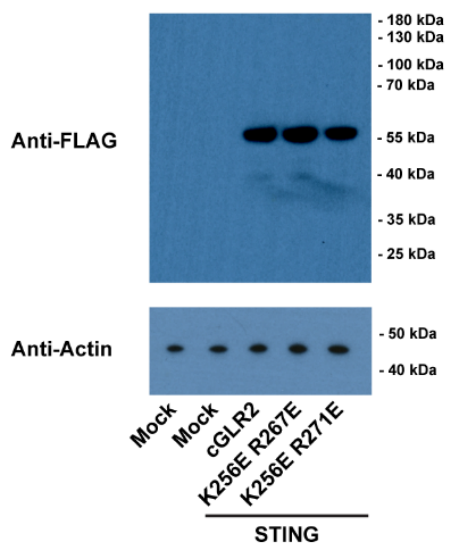

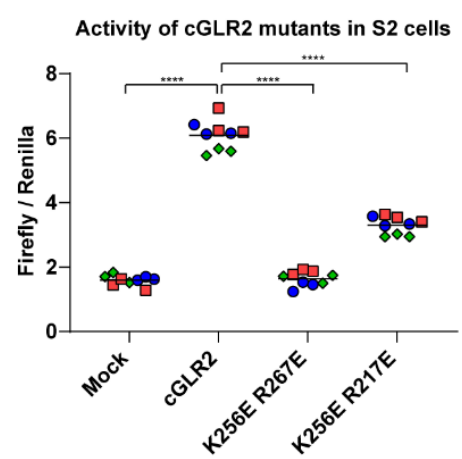

\section{Extended Data Fig. 11 | cGLR1 produces 3'2'-cGAMP in response to dsRNA. a,} Representative chromatograms from anion exchange chromatography analysis of different nucleotides. b, Representative chromatograms from anion exchange chromatography analysis of reaction products from activity assays with recombinant cGLR1 in the presence of different nucleic acids. c, Immunoblot showing the expression of FLAG-tagged cGLR2 and mutants thereof from Fig. 3f. d, S2 cells transfected with cGLR2 or mutants thereof as well as plasmids encoding firefly or Renilla luciferase under transcriptional control of the Sting or Actin5C promoter, respectively. Data are from three independent experiments (blue, red, and green, each performed in triplicates) and shown with mean $(n=9)$. In $\mathbf{d}$, data were analyzed using two-way ANOVA and Holm-Š́dák post hoc test and compared to cGLR2. ns not significant, $* * * * P<0.0001$. 


\section{References}

1 Whiteley, A. T. et al. Bacterial cGAS-like enzymes synthesize diverse nucleotide signals. Nature 567, 194-199, doi:10.1038/s41586-019-0953-5 (2019).

2 Ablasser, A. et al. cGAS produces a 2'-5'-linked cyclic dinucleotide second messenger that activates STING. Nature 498, 380-384, doi:10.1038/nature12306 (2013).

3 Civril, F. et al. Structural mechanism of cytosolic DNA sensing by cGAS. Nature 498, 332-337, doi:10.1038/nature12305 (2013).

4 Sun, L., Wu, J., Du, F., Chen, X. \& Chen, Z. J. Cyclic GMP-AMP synthase is a cytosolic DNA sensor that activates the type I interferon pathway. Science 339, 786791, doi:10.1126/science.1232458 (2013).

$5 \mathrm{Wu}$, J. et al. Cyclic GMP-AMP is an endogenous second messenger in innate immune signaling by cytosolic DNA. Science 339, 826-830, doi:10.1126/science.1229963 (2013).

6 Lowey, B. et al. CBASS Immunity Uses CARF-Related Effectors to Sense 3'-5'- and 2'-5'-Linked Cyclic Oligonucleotide Signals and Protect Bacteria from Phage Infection. Cell 182, 38-49 e17, doi:10.1016/j.cell.2020.05.019 (2020).

7 Morehouse, B. R. et al. STING cyclic dinucleotide sensing originated in bacteria. Nature 586, 429-433, doi:10.1038/s41586-020-2719-5 (2020).

8 Cohen, D. et al. Cyclic GMP-AMP signalling protects bacteria against viral infection. Nature 574, 691-695, doi:10.1038/s41586-019-1605-5 (2019).

9 Guo, Z., Li, Y. \& Ding, S. W. Small RNA-based antimicrobial immunity. Nature reviews. Immunology 19, 31-44, doi:10.1038/s41577-018-0071-x (2019).

10 Schneider, J. \& Imler, J. L. Sensing and signalling viral infection in drosophila. Dev Comp Immunol 117, 103985, doi:10.1016/j.dci.2020.103985 (2021).

11 Goto, A. et al. The Kinase IKKbeta Regulates a STING- and NF-kappaB-Dependent Antiviral Response Pathway in Drosophila. Immunity 49, 225-234 e224, doi:10.1016/j.immuni.2018.07.013 (2018).

12 Liu, Y. et al. Inflammation-Induced, STING-Dependent Autophagy Restricts Zika Virus Infection in the Drosophila Brain. Cell Host Microbe 24, 57-68 e53, doi:10.1016/j.chom.2018.05.022 (2018).

13 Hua, X. et al. Stimulator of interferon genes (STING) provides insect antiviral immunity by promoting Dredd caspase-mediated NF-kappaB activation. J Biol Chem 293, 11878-11890, doi:10.1074/jbc.RA117.000194 (2018).

14 Donelick, H. M. et al. In vitro studies provide insight into effects of Dicer-2 helicase mutations in Drosophila melanogaster. RNA 26, 1847-1861, doi:10.1261/rna.077289.120 (2020).

15 Jin, L. et al. MPYS is required for IFN response factor 3 activation and type I IFN production in the response of cultured phagocytes to bacterial second messengers cyclic-di-AMP and cyclic-di-GMP. J Immunol 187, 2595-2601, doi:10.4049/jimmunol.1100088 (2011).

16 Burdette, D. L. et al. STING is a direct innate immune sensor of cyclic di-GMP. Nature 478, 515-518, doi:10.1038/nature10429 (2011).

17 Diner, E. J. et al. The innate immune DNA sensor cGAS produces a noncanonical cyclic dinucleotide that activates human STING. Cell Rep 3, 1355-1361, doi:10.1016/j.celrep.2013.05.009 (2013).

18 Gao, P. et al. Cyclic $\left[\mathrm{G}\left(2^{\prime}, 5^{\prime}\right) \mathrm{pA}\left(3^{\prime}, 5^{\prime}\right) \mathrm{p}\right]$ is the metazoan second messenger produced by DNA-activated cyclic GMP-AMP synthase. Cell 153, 1094-1107, doi:10.1016/j.cell.2013.04.046 (2013). 
19 Kranzusch, P. J., Lee, A. S., Berger, J. M. \& Doudna, J. A. Structure of human cGAS reveals a conserved family of second-messenger enzymes in innate immunity. Cell Rep 3, 1362-1368, doi:10.1016/j.celrep.2013.05.008 (2013).

20 Zhang, X. et al. Cyclic GMP-AMP containing mixed phosphodiester linkages is an endogenous high-affinity ligand for STING. Mol Cell 51, 226-235, doi:10.1016/j.molcel.2013.05.022 (2013).

21 Cai, H. et al. 2'3'-cGAMP triggers a STING- and NF-kappaB-dependent broad antiviral response in Drosophila. Sci Signal 13, doi:10.1126/scisignal.abc4537 (2020).

$22 \mathrm{Wu}, \mathrm{X}$. et al. Molecular evolutionary and structural analysis of the cytosolic DNA sensor cGAS and STING. Nucleic Acids Res 42, 8243-8257, doi:10.1093/nar/gku569 (2014).

23 Martin, M., Hiroyasu, A., Guzman, R. M., Roberts, S. A. \& Goodman, A. G. Analysis of Drosophila STING Reveals an Evolutionarily Conserved Antimicrobial Function. Cell Rep 23, 3537-3550 e3536, doi:10.1016/j.celrep.2018.05.029 (2018).

24 Caygill, E. E. \& Brand, A. H. The GAL4 System: A Versatile System for the Manipulation and Analysis of Gene Expression. Methods Mol Biol 1478, 33-52, doi:10.1007/978-1-4939-6371-3_2 (2016).

25 Tanaka, Y. \& Chen, Z. J. STING specifies IRF3 phosphorylation by TBK1 in the cytosolic DNA signaling pathway. Sci Signal 5, ra20, doi:10.1126/scisignal.2002521 (2012).

26 Zhong, B. et al. The adaptor protein MITA links virus-sensing receptors to IRF3 transcription factor activation. Immunity 29, 538-550, doi:10.1016/j.immuni.2008.09.003 (2008).

27 Palmer, W. H., Medd, N. C., Beard, P. M. \& Obbard, D. J. Isolation of a natural DNA virus of Drosophila melanogaster, and characterisation of host resistance and immune responses. PLoS Pathog 14, e1007050, doi:10.1371/journal.ppat. 1007050 (2018).

28 Slavik, K. M. et al. cGAS-like receptors control RNA sensing and 3'2'-cGAMP antiviral signaling in Drosophila. Under consideration at Nature (2021).

29 Hartmann, R., Justesen, J., Sarkar, S. N., Sen, G. C. \& Yee, V. C. Crystal structure of the 2'-specific and double-stranded RNA-activated interferon-induced antiviral protein 2'-5'-oligoadenylate synthetase. Mol Cell 12, 1173-1185, doi:10.1016/s10972765(03)00433-7 (2003).

30 Gui, X. et al. Autophagy induction via STING trafficking is a primordial function of the cGAS pathway. Nature 567, 262-266, doi:10.1038/s41586-019-1006-9 (2019).

31 McFarland, A. P. et al. Sensing of Bacterial Cyclic Dinucleotides by the Oxidoreductase RECON Promotes NF-kappaB Activation and Shapes a Proinflammatory Antibacterial State. Immunity 46, 433-445, doi:10.1016/j.immuni.2017.02.014 (2017).

32 Eaglesham, J. B., McCarty, K. L. \& Kranzusch, P. J. Structures of diverse poxin cGAMP nucleases reveal a widespread role for cGAS-STING evasion in hostpathogen conflict. Elife 9, doi:10.7554/eLife.59753 (2020).

33 Eaglesham, J. B., Pan, Y., Kupper, T. S. \& Kranzusch, P. J. Viral and metazoan poxins are cGAMP-specific nucleases that restrict cGAS-STING signalling. Nature 566, 259-263, doi:10.1038/s41586-019-0928-6 (2019).

34 Hernaez, B. et al. Viral cGAMP nuclease reveals the essential role of DNA sensing in protection against acute lethal virus infection. Sci $A d v$ 6, doi:10.1126/sciadv.abb4565 (2020).

35 Lowey, B. \& Kranzusch, P. J. CD-NTases and nucleotide second messenger signaling. Curr Biol 30, R1106-R1108, doi:10.1016/j.cub.2020.06.096 (2020). 
36 Andersen, L. L. et al. Frequently used bioinformatics tools overestimate the damaging effect of allelic variants. Genes Immun 20, 10-22, doi:10.1038/s41435-017-0002-z (2019).

37 Andersen, L. L. et al. Functional IRF3 deficiency in a patient with herpes simplex encephalitis. J Exp Med 212, 1371-1379, doi:10.1084/jem.20142274 (2015).

38 Schulz, A., Jankowski, V., Zidek, W. \& Jankowski, J. Highly sensitive, selective and rapid LC-MS method for simultaneous quantification of diadenosine polyphosphates in human plasma. J Chromatogr B Analyt Technol Biomed Life Sci 961, 91-96, doi:10.1016/j.jchromb.2014.05.018 (2014).

39 Wang, Y., Holleufer, A., Gad, H. H. \& Hartmann, R. Length dependent activation of OAS proteins by dsRNA. Cytokine 126, 154867, doi:10.1016/j.cyto.2019.154867 (2020).

40 Holleufer, A. \& Hartmann, R. A Highly Sensitive Anion Exchange Chromatography Method for Measuring cGAS Activity in vitro. Bio-protocol 8, e3055, doi:10.21769/BioProtoc.3055 (2018). 